INRA Prod. Anim., 2011, 24 (5), 415-432

\title{
Les gaz à effet de serre en élevage bovin : évaluation et leviers d'action
}

\author{
J.-B. DOLLE' 1 , J. AGABRIEL ${ }^{2}$, J.-L. PEYRAUD 3,4, P. FAVERDIN 3,4, V. MANNEVILLE ${ }^{5}$, \\ C. RAISON ${ }^{6}, A$. GAC6 6 , A. LE GALL 6 \\ ${ }^{1}$ Institut de l'Elevage, 56 Avenue Roger Salengro, BP 80039, F-62051 Saint-Laurent-Blangy, cedex, France \\ 2 INRA, UR1213 Herbivores, F-63122 Saint-Genès-Champanelle, France \\ ${ }^{3}$ INRA, UMR1080 Production du Lait, Domaine de la Prise, F-35590 Saint-Gilles, France \\ ${ }_{4}$ Agrocampus Ouest, Production du lait, F-35590 Saint-Gilles, France \\ 5 Institut de l'Elevage, 9 Allée Pierre de Fermat, F-63170 Aubière, France \\ ${ }^{6}$ Institut de l'Elevage, Monvoisin, BP 85225, F-35652 Le-Rheu, France \\ Courriel : jean-baptiste.dolle@idele.fr
}

L'élevage bovin contribue pour près de $60 \%$ aux émissions de gaz à effet de serre du secteur agricole. Cet article fait le point sur les méthodes d'évaluation de l'empreinte carbone de la production de lait et de viande, et en analyse les principaux facteurs de variation afin d'identifier les leviers d'action et les marges de progrès.

En 2006 le rapport de la FAO «Livestock long shadow : environnemental issues and options» évaluait la contribution mondiale de l'élevage aux émissions de Gaz à Effet de Serre (GES) à 18\% (FAO 2006). Des travaux plus récents ont révisé les valeurs de certains secteurs comme l'élevage laitier. Dans le cadre de ces nouvelles évaluations des émissions de GES à l'échelle mondiale conduites en 2010, la contribution du secteur bovin laitier est évaluée à 2,7\% (FAO 2010). Ces évaluations menées par la FAO concernent toute la chaîne de production, intégrant la production des aliments animaux, la gestion des troupeaux, les déjections, le transport du lait, etc. En France, l'activité agricole représentait 18,8\% des émissions de GES nationales en 2009 (hors $\mathrm{CO}_{2}$ fossile intégré dans le secteur des transports), dont $10 \%$ liés directement aux exploitations bovines, soit près de $60 \%$ des émissions agricoles, en considérant les surfaces affectées à l'élevage (CITEPA 2011). A l'échelle européenne, une étude basée sur une analyse du cycle de vie jusqu'au portail de la ferme a été conduite par le Joint Research Center (JRC) (Leip et al 2010). Cette étude met en avant une contribution du secteur de l'élevage herbivore et granivore aux émissions de GES à hauteur de 9,1\% sans considérer le changement d'occupation des sols (déforestation, retournement de prairies) et à hauteur de $12,8 \%$ en intégrant le changement d'occupation des sols. Les cheptels laitier et à viande contribuent chacun à hauteur de $29 \%$ des émissions, la production porcine à hauteur de $25 \%$ et les autres productions à hauteur de $17 \%$ (Leip et al 2010).

Les processus conduisant aux émissions de GES en élevage sont complexes et imbriqués. On considère que toutes les émissions de gaz carbonique $\left(\mathrm{CO}_{2}\right)$ et d'eau liées à la respiration des végétaux et des animaux sont «biogéniques» et ne constituent pas une contribution additionnelle à l'effet de serre. En revanche, le protoxyde d'azote $\left(\mathrm{N}_{2} \mathrm{O}\right)$ et le méthane $\left(\mathrm{CH}_{4}\right)$, de même que le gaz carbonique $\left(\mathrm{CO}_{2}\right)$ issu de la combustion des carburants fossiles, sont «anthropiques» et doivent à ce titre être gérés comme les émissions des autres activités humaines.

Pour réduire l'effet de l'activité anthropique sur le changement climatique, le protocole de Kyoto, ratifié en 2005, visait une réduction des émissions de GES au niveau mondial de $5,2 \%$ en 2012 par rapport à 1990 , soit $8 \%$ pour l'Union Européenne et une stabilisation pour la France. Dans le cadre d'un second engagement post 2012, les objectifs de réduction pourraient être portés à 20 voire $30 \%$ d'ici 2020 par rapport à 1990. Dans le cadre du «Paquet énergie climat», les politiques européennes emboîtent le pas du proto- cole de Kyoto avec un objectif affiché de réduction des émissions des GES de $20 \%$ à l'horizon 2020 par rapport à 1990, voire $30 \%$ dans le cas d'un accord international. Face à ces objectifs, les états membres de l'Union Européenne mettent en place des politiques destinées à intégrer les aspects environnementaux dans les actions de production et de consommation. Ainsi, dans le cadre du Grenelle de l'Environnement, la France envisage d'adopter des modes de production et de consommation durables. Les lois Grenelle 1 et 2 ambitionnent le développement de mesures en faveur de la consommation durable comme la réduction des émissions de GES et des consommations d'énergie, et l'affichage des impacts environnementaux des produits de grande consommation. En Allemagne, dans un rapport publié en 2010, le gouvernement met en avant les mesures permettant de réduire les émissions de GES. Aux Pays-Bas, le Ministère de l'Agriculture a signé un engagement avec les principaux représentants du secteur agricole pour une réduction de $30 \%$ des émissions de GES agricoles entre 1990 et 2020 au moyen de politiques volontaristes. Une action similaire est conduite au Royaume-Uni, avec la rédaction de feuilles de route sur la réduction des émissions de GES associant les éleveurs, les industriels et les consommateurs. Parallèlement, des initiatives privées sur l'évaluation de 
l'empreinte carbone des produits de grande consommation voient le jour. Ces évaluations doivent informer les instances administratives et les consommateurs de l'impact potentiel des produits, notamment alimentaires.

En élevage de ruminants, l'appréciation analytique des émissions par gaz ou par poste revêt un intérêt pour l'acquisition de connaissances sur les mécanismes d'émission. Cependant, la complexité des processus et les interactions entre les différents composants que sont le sol, les prairies, les cultures, les aliments, les animaux, etc... nécessitent également de prendre en compte le mécanisme de stockage de carbone associé à la présence de prairies. Cette problématique des émissions de GES et du stockage de carbone pose ainsi la question du type d'approche à considérer, approche sectorielle vs approche globale. Les investigations conduites ces dernières années mettent en avant la nécessité d'élaborer des démarches d'évaluation globale et non uniquement focalisées sur un gaz ou une pratique. Une évaluation globale permet ainsi de considérer l'ensemble des flux présents sur une exploitation et entre les différents compartiments du système (solanimal-plante). Sur cette base, il apparaît essentiel de développer des évaluations globales à l'échelle des systèmes de production et d'approfondir les connaissances sur les facteurs explicatifs de la variabilité des impacts environnementaux observés et de positionner les pratiques à mettre en œuvre dans les exploitations bovines. La méthodologie mobilisée est basée sur l'Analyse du Cycle de Vie (ACV). Des choix méthodologiques doivent être opérés afin d'adapter cette méthode, initialement développée pour les produits industriels, aux produits agricoles. Les fondements méthodologiques de cette approche se trouvent améliorés par des travaux de recherche ou d'évaluation permettant de préciser les facteurs d'émission de GES et de stockage de carbone, le périmètre de l'évaluation, les allocations entre coproduits, etc. Appliquée à des systèmes de production contrastés et à des situations présentant des niveaux d'optimisation différents, elle permet d'identifier les leviers d'action et d'en apprécier leur efficacité.

\section{1 / Les éléments méthodolo- giques mis en ouvre pour l'évaluation de l'impact GES}

\section{1 / Une méthodologie basée sur l'ACV}

Plusieurs gaz sont responsables de l'accroissement de l'effet de serre : le dioxyde de carbone $\left(\mathrm{CO}_{2}\right)$, le méthane $\left(\mathrm{CH}_{4}\right)$, le protoxyde d'azote $\left(\mathrm{N}_{2} \mathrm{O}\right)$ pour les principaux mais aussi d'autres gaz comme les gaz fluorés (CFC, HFC, $\mathrm{PFC}, \mathrm{SF}_{6}$ ), qui ne concernent pas le domaine agricole. La contribution à l'effet de serre des différents gaz est variable et s'exprime par leur Potentiel de Réchauffement Global (PRG). Le PRG d'un gaz se définit comme le forçage radiatif, cumulé sur une durée définie, ici de 100 ans. Cette valeur se mesure relativement au $\mathrm{CO}_{2}$ et l'impact sur l'effet de serre de chacun des gaz s'exprime en kilogramme d'équivalent $\mathrm{CO}_{2}\left(25 \mathrm{~kg} \mathrm{CO} / \mathrm{kg}\right.$ de $\mathrm{CH}_{4}$ et $298 \mathrm{~kg}$ $\mathrm{CO}_{2} / \mathrm{kg}$ de $\mathrm{N}_{2} \mathrm{O}$ ). Les émissions de GES sont ensuite exprimées en lien avec la fonction primaire représentée par le produit. Pour une approche produit/ filière, l'Unité Fonctionnelle (UF) est généralement la quantité de produit, exprimée souvent en poids ou unité de volume ( $\mathrm{kg}$ de produit brut, $\mathrm{kg}$ de matière sèche, $\mathrm{kg}$ de viande, litre de lait). Il peut également s'agir d'une unité relative à un critère de qualité ou à une fonctionnalité recherchée (kg de protéines, kcal d'énergie brute, MJ d'énergie potentielle...).

Cette évaluation de l'impact environnemental «GES» est classiquement réalisée en s'appuyant sur la méthode de l'Analyse du Cycle de Vie, relevant de la norme 14040 . Celle-ci permet ainsi d'observer les impacts environnementaux potentiels directs et indirects d'un produit tout au long de son cycle de vie. Initialement mise en œuvre pour des produits manufacturés, l'application de l'ACV au secteur agricole se développe (Rossier et Gaillard 2001). D'importantes études scientifiques ont ainsi été conduites au niveau mondial (FAO 2006, 2010), européen (Leip et al 2010), national (Garnett 2007, Kool et al 2009) et à l'échelle du produit agricole (Cederberg et Flysjö 2004, BassetMens et al 2005, Van der Werf et al 2009, Veysset et al 2010, Kristensen et al 2011) dans le but d'appréhender l'impact du secteur agricole et de l'élevage herbivore.

La mise en œuvre de l'ACV nécessite le recours à des facteurs d'émission qui indiquent la quantité de GES émise par un processus ou une activité. Pour cela, le Groupement Intergouvernemental d'Experts sur l'Evolution du Climat (GIEC) propose une évaluation selon trois niveaux de précision croissant (IPCC 2006) :

- Niveau 1 (dit Tier 1), qui propose des données et des équations par défaut, non spécifiques au pays ;

- Niveau 2 (dit Tier 2), qui propose d'appliquer des coefficients basés sur des données spécifiques au pays ou à la région (données d'activités plus détaillées, coefficients spécifiques au pays) ;

- Niveau 3 (dit Tier 3), qui propose d'utiliser des modèles et des systèmes de mesures d'inventaire adaptés aux conditions nationales, (données d'activité à résolution plus élevée, échelles spatiale et temporelle supérieures) permettant d'aboutir à des estimations plus précises et prenant mieux en compte les options de mitigation mises en œuvre pour réduire ces émissions. Ces modèles doivent cependant utiliser des données accessibles au niveau national pour calculer les inventaires. Elles doivent aussi être reconnues par une instance internationale faute de quoi les résultats ne peuvent pas être pris en considération par les décideurs publics.

Fidèle à la méthodologie ACV et aux préconisations du GIEC, la Fédération Internationale Laitière (FIL) a ainsi publié une méthodologie de niveau 2 adaptée au secteur laitier (FIL 2010). D'autres initiatives internationales sont en cours, notamment pour la production d'agneaux. En France, les Instituts Techniques Agricoles ont développé la méthode GES'TIM (niveau 3) qui propose des méthodes d'estimation et facteurs d'émission spécifiques au territoire français : conditions pédoclimatiques, pratiques d'élevage et itinéraires culturaux, bouquet énergétique français, provenance et itinéraires de production des intrants adaptés à l'approvisionnement des exploitations françaises ( $\mathrm{Gac}$ et al 2010a).

\section{2 / Le périmètre d'analyse et les sources d'émission}

En exploitation agricole, l'application de la méthodologie ACV revient à considérer l'ensemble des impacts sur la partie du cycle de vie du produit s'arrêtant au portail de la ferme. Cette évaluation constitue ainsi un premier maillon à l'évaluation globale des GES du produit incluant l'amont agricole, le transport, la transformation, la distribution, etc. Sur le périmètre s'arrêtant au portail de l'exploitation, il s'agit d'inventorier les impacts directs liés au processus de production au niveau de l'exploitation agricole mais également les impacts indirects inhérents à la fabrication des intrants et à leur transport (figure 1). Cela concerne généralement les animaux en production, les animaux de renouvellement, les surfaces destinées à l'atelier, et l'ensemble des intrants (énergie, fertilisants, alimentation...) de cet atelier et de ces surfaces (Gac et al 2010c). Pour le lait et la viande consommés, les émissions associées au périmètre de l'exploitation représentent 70 à $90 \%$ des émissions totales de l'ensemble du cycle de vie des deux 
Figure 1. Postes d'émissions et flux de matières retenus pour l'évaluation de l'impact «gaz à effet de serre».

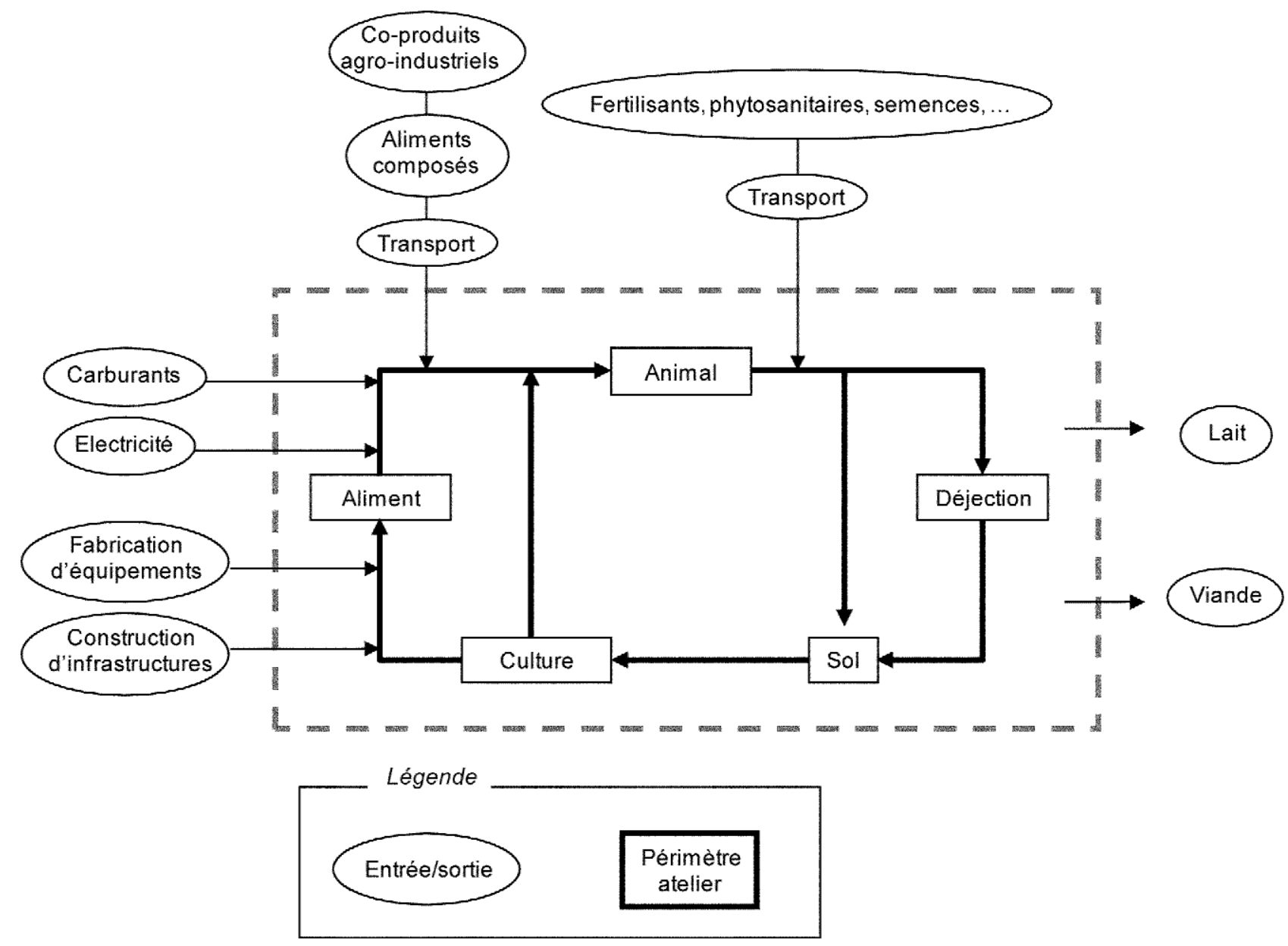

produits (Tomasula et Nutter 2011). Une bonne connaissance des impacts à l'échelle de l'exploitation est donc essentielle pour l'évaluation de l'impact sur le cycle de vie complet des produits consommés en alimentation humaine.

Les sources d'émission associées à ce périmètre sont regroupées en cinq postes principaux :

1/ La fermentation entérique : émissions de méthane issues de l'activité biologique des animaux de l'atelier;

2/ La gestion des déjections : émissions de méthane et de protoxyde d'azote issues de la gestion des déjections (pâturage, bâtiment, stockage) ;

3/ Les apports azotés : émissions de protoxyde d'azote liées aux phénomènes de nitrification et dénitrification des apports directs d'azote par la voie de la fertilisation organique (y compris résidus de culture) et minérale, et des apports indirects résultant de l'enrichissement en azote par le lessivage de nitrates et la volatilisation d'ammoniac ;

4/ L'énergie directe: émissions de $\mathrm{CO}_{2}$ résultant des énergies fossiles consommées sur l'exploitation (électricité et fioul);

5/ Les intrants : impact en équivalent $\mathrm{CO}_{2}$ généré lors de la fabrication et du transport des intrants (engrais, aliments du bétail, semences). Il s'agit en particulier de $\mathrm{CO}_{2}$ d'origine énergétique, mais aussi des autres GES, dont le $\mathrm{N}_{2} \mathrm{O}$ issu de la fabrication des engrais.

Les facteurs des principaux postes d'émission mobilisés pour l'évaluation des systèmes d'élevage herbivore français sont issus de la méthode GES'TIM (tableau 1). Les bâtiments et matériels, qui représentent moins de $5 \%$ de l'impact GES, peuvent être retenus dans le champ de l'étude (FIL 2010), mais sont souvent exclus compte tenu des faibles différences observées entre les techniques constructives (Erzinger et al 2003) et du faible impact GES par rapport aux émissions totales (Blanchin et al 2010). Les émissions associées aux pesticides, détergents, produits vétérinaires dont l'impact GES est inférieur à $1 \%$ sont généralement exclus du périmètre (Henriksson et al 2011).

\section{3 / Le changement d'utilisation des sols et la séquestration du carbone}

Au-delà des émissions de GES attachées aux processus de production, les flux de carbone liés ou non à un changement d'utilisation des sols doivent également être considérés dans l'évaluation de l'impact GES. On parle de changement d'utilisation des sols lorsqu'un sol non agricole est transformé en sol agricole ou lorsqu'un changement notoire d'utilisation agricole est intervenu. Ces émissions de GES ou séquestration de carbone associées respectivement au destockage ou au stockage de carbone doivent ainsi être évaluées pour chacune des entrées du cycle de vie du produit. C'est notamment le cas des aliments importés qui entrent dans la composition des rations et qui sont associés à une conversion des terres, comme le soja. Ainsi pour le tourteau de soja, les données retenues par la FAO (2010) $(0,93 \mathrm{~kg} \mathrm{CO} / \mathrm{kg}$ de tourteau de soja provenant d'Argentine partiellement associé à un retournement des prairies en culture et $7,69 \mathrm{~kg} \mathrm{CO} / \mathrm{kg}$ pour du tourteau de soja provenant du Brésil et 
Tableau 1. Principaux postes et facteurs d'émission - méthode GES'TIM (Gac et al 2010).

\begin{tabular}{|c|c|c|}
\hline $\mathrm{CH}_{4}$ entérique & $\begin{array}{l}113,5 \mathrm{~kg} \mathrm{CH}_{4} / \mathrm{an} / \mathrm{VL} \text { à } 5900 \mathrm{~kg} \text { lait } \\
131,2 \mathrm{~kg} \mathrm{CH}_{4} / \mathrm{an} / \mathrm{VL} \text { à } 7700 \mathrm{~kg} \text { lait }\end{array}$ & $72,0 \mathrm{~kg} \mathrm{CH}_{4} / \mathrm{an} / \mathrm{Vache}$ allaitante \\
\hline $\mathrm{CH}_{4}$ pâturage & \multicolumn{2}{|l|}{$0,8 \mathrm{~g} \mathrm{CH}_{4} / \mathrm{UGB} / \mathrm{j}$} \\
\hline $\mathrm{CH}_{4}$ déjections au bâtiment & \multicolumn{2}{|c|}{$\begin{array}{l}81 \mathrm{~kg} \mathrm{CH} / \mathrm{UGB} / a n \text { en litière accumulée } \\
6,7 \mathrm{~kg} \mathrm{CH}_{4} / \mathrm{UGB} / a n \text { en stabulation entravée, aire raclée } \\
36,5 \mathrm{~kg} \mathrm{CH}_{4} / \mathrm{UGB} / a n \text { en litière accumulée et aire raclée } \\
111 \mathrm{~kg} \mathrm{CH} / \mathrm{UGB} / a n \text { en caillebotis intégral }\end{array}$} \\
\hline $\mathrm{CH}_{4}$ stockage déjections & \multicolumn{2}{|l|}{$\begin{array}{l}13 \mathrm{~kg} \mathrm{CH} / \mathrm{an} / \mathrm{m}^{3} \text { de lisier } \\
4,5 \mathrm{~kg} \mathrm{CH} / \mathrm{an} / \text { tonne de fumier }\end{array}$} \\
\hline $\mathrm{N}_{2} \mathrm{O}$ déjections au bâtiment & \multicolumn{2}{|c|}{$\begin{array}{l}0,26 \mathrm{~kg} \mathrm{~N} \mathrm{~N}_{2} \mathrm{O} / \mathrm{UGB} / \mathrm{an} \text { en litière accumulée } \\
0,0 \mathrm{~kg} \mathrm{~N} \mathrm{~N}_{2} \mathrm{O} / \mathrm{UGB} / \mathrm{an} \text { en stabulation entravée, aire raclée } \\
0,10 \mathrm{~kg} \mathrm{~N} \mathrm{~N}_{2} \mathrm{O} / \mathrm{UGB} / \mathrm{an} \text { en litière accumulée et aire raclée } \\
0,17 \mathrm{~kg} \mathrm{~N} N_{2} \mathrm{O} / \mathrm{UGB} / \mathrm{an} \text { en caillebotis intégral }\end{array}$} \\
\hline $\mathrm{N}_{2} \mathrm{O}$ stockage déjections & \multicolumn{2}{|l|}{$\begin{array}{l}0 \mathrm{~g} \mathrm{~N}_{2} \mathrm{O} / \mathrm{kg} \mathrm{N} \text { stocké en lisier } \\
4,7 \mathrm{~g} \mathrm{~N}_{2} \mathrm{O} / \mathrm{kg} \mathrm{N} \text { stocké en fumier }\end{array}$} \\
\hline $\mathrm{N}_{2} \mathrm{O}$ excrétion au pâturage & \multicolumn{2}{|c|}{$\left(1,5 \%\right.$ Nurine $+0,4 \%$ Nfèces) $\times 1,5 \times 44 / 28 \mathrm{~kg} \mathrm{~N} \mathrm{~N}_{2} \mathrm{O} / \mathrm{ha} / \mathrm{an}$} \\
\hline $\mathrm{N}_{2} \mathrm{O}$ épandage & \multicolumn{2}{|c|}{$\begin{array}{l}1 \% \text { du } N \text { apporté (épandage organique ou minéral, résidus de culture) } \\
0 \% \text { du } N \text { fixé par les légumineuses } \\
1 \% \text { du } N \text { volatilisé } \\
0,75 \% \text { du } N \text { lessivé }\end{array}$} \\
\hline $\mathrm{CO}_{2}$ & \multicolumn{2}{|c|}{$\begin{array}{l}3,06 \mathrm{~kg} \mathrm{CO} / \mathrm{litre} \text { de fioul consommé sur l'exploitation } \\
6,1 \mathrm{~kg} \text { eq. } \mathrm{CO}_{2} / \mathrm{kg} \mathrm{N} \text { minéral importé } \\
1,6 \mathrm{~kg} \text { eq. } \mathrm{CO}_{2} / \mathrm{kg} \text { tourteau soja importé }\end{array}$} \\
\hline
\end{tabular}

totalement associé à de la déforestation) ou celles déterminées par Da Silva et al (2010) $(0,69 \mathrm{~kg} \mathrm{CO} / \mathrm{kg}$ de tourteau de soja provenant du centre est du Brésil et $0,34 \mathrm{~kg} \mathrm{CO} / \mathrm{kg}$ de tourteau de soja provenant du sud du Brésil) sont très disparates. Ces différences liées à des périmètres d'évaluation et une allocation de l'impact différent, mettent en évidence l'importance que représente la prise en compte de ces flux et la nécessité d'en préciser les valeurs. Au-delà des produits importés, la séquestration du carbone concerne également les surfaces nationales ayant fait l'objet d'un changement d'usage des terres. Une expertise collective menée par l'INRA (Arrouays et al 2002) a ainsi permis de mettre en évidence les niveaux moyens de stockage/déstockage observés en France (tableau 2) suite à une conversion des terres. Le cas des prairies est tout particulièrement important compte tenu de leur place dans l'élevage français. Le retournement ou l'implantation de prairies affectent les flux de carbone. Ainsi, la conversion d'une forêt en prairies ou en cultures, ou d'une prairie en cultures, se traduit par des émissions de gaz carbonique allant jusqu'à 4,6 tonnes $\mathrm{CO}_{2}$ /ha/an et de protoxyde d'azote associées à une décomposition de la matière organique contenue dans les sols. Inversement, le passage d'un sol cultivé à une prairie se traduit par une séquestration du carbone dans les sols représentant de 0,84 à 2,75 tonnes $\mathrm{CO}_{2} / \mathrm{ha} / \mathrm{an}$ (Arrouays et al 2002).

Par ailleurs, dans des situations «stabilisées» sans changement notoire d'utilisation des sols, certains auteurs (Watson et al 2002) spécifient que la teneur en matière organique du sol atteint un équilibre y compris sur des sols prairiaux où le flux annuel serait nul. Néanmoins, plusieurs publications (Bellamy et al 2005, Smith et al 2007, Soussana et Lüscher 2007) mettent en évidence la présence d'un stockage annuel de carbone pour des surfaces en prairies pendant une longue durée. Il n'y aurait ainsi pas de limite temporelle au stockage du carbone, des prairies très anciennes pouvant continuer à stocker du carbone sur de très longues périodes.
Des travaux récents sur le stockage de carbone sous prairies menés dans le cadre des projets européens GreenGrass (Soussana et al 2007) et CarboEurope (Schulze et al 2009) montrent par ailleurs que la productivité primaire brute des prairies est comparable à celle des forêts en Europe. Les prairies constituent des puits nets pour le $\mathrm{CO}_{2}$ atmosphérique, stockant de 500 à 1200 $\mathrm{kg} \mathrm{C} /$ ha/an selon les modalités de gestion (chargement animal, mode d'utilisation, fertilisation). Aux Etats-Unis, Pelletier et al (2010) proposent des valeurs de 120 à $400 \mathrm{~kg} \mathrm{C} / \mathrm{ha} / \mathrm{an}$ sur prairies. Ce stockage de carbone dans les sols peut toutefois présenter une certaine vulnérabilité au changement climatique (Ciais et al 2003), notamment en raison de l'augmentation de la température favorable à la minéralisation du carbone stocké et donc au recyclage de gaz carbonique. Néanmoins, ce phénomène potentiel de déstockage peut être compensé par l'augmentation du $\mathrm{CO}_{2}$ atmosphérique qui réduit la sensibilité des écosystèmes prairiaux à la sécheresse (Morgan et al 2004) et par la 
Tableau 2. Impacts liés au changement d'usage des terres sur le stockage de carbone dans les sols (Arrouays et al 2002).

\begin{tabular}{|l|c|c|}
\hline Conversion des terres & $\begin{array}{c}\text { Flux additionnel annuel moyen } \\
\text { en T C/ha/an (scénario à } 20 \text { ans) }\end{array}$ & $\begin{array}{c}\text { Equivalences en } \mathrm{CO}_{2} \text { émis ou } \\
\text { capté }\left(\mathrm{T} \text { eq. } \mathrm{CO}_{2} / \text { ha/an) }\right.\end{array}$ \\
\hline Stockage de carbone & Captage de $\mathrm{CO}_{2}$ \\
\hline Culture $\rightarrow$ Prairie permanente & $0,49 \pm 0,26$ & 0,84 à 2,75 \\
\hline Culture $\rightarrow$ Boisement & $0,44 \pm 0,24$ & 0,73 à 2,49 \\
\hline Prairie permanente $\rightarrow$ Boisement & Inférieur à $+0,1 \pm 0,2$ & $-0,1$ à 0,3 \\
\hline & Déstockage de carbone & Em issions de $\mathrm{CO}_{2}$ \\
\hline Prairie permanente $\rightarrow$ Culture & $-0,95 \pm 0,3$ & 2,4 à 4,6 \\
\hline Bois $\rightarrow$ Culture & $-0,75$ & 2,75 \\
\hline Bois $\rightarrow$ Prairie permanente & $-0,1 \pm 0,1$ & 0 à 0,7 \\
\hline
\end{tabular}

productivité des prairies qui augmente de 5 à $15 \%$ selon la disponibilité en eau et en substances nutritives (Soussana et Hartwig 1996, Tubiello et al 2007).

Pour apprécier la séquestration du carbone, la méthodologie IPCC (2006) recommande de considérer les pertes et les gains de carbone observés durant les 20 premières années suivant le changement d'utilisation du sol. Compte tenu de l'absence de données à l'échelle mondiale, les évaluations et les méthodologies développées pour la détermination des GES (FIL 2010, FAO 2010) ne considèrent pas le stockage de carbone des systèmes prairiaux de plus de 20 ans. Au niveau européen, le JRC retient des niveaux de stockage de $237 \mathrm{~kg} \mathrm{C} / \mathrm{ha}$ pour des prairies permanentes et $115 \mathrm{~kg}$ $\mathrm{C} /$ ha pour des prairies temporaires (Leip et al 2010). Dans un contexte où ce point est discuté, des facteurs moyens de stockage/déstockage (Gac et al 2010b) ont été proposés pour les systèmes herbivores français (tableau 3 ). Ces niveaux de stockage proposés sont volontairement prudents au regard des dernières références publiées et citées précédemment. Dans l'attente de travaux complémentaires provenant de programmes financés par l'UE pour mieux les préciser, ils constituent une valeur moyenne au regard de la variabilité des situations (plaine, montagne, âge de la prairie...) et des niveaux d'incertitude associés. Enfin, il ne faut pas négliger le stockage de carbone sous les haies et les bosquets, très fréquemment rencontrés dans les exploitations d'élevage. Leur surface, qui peut représenter entre 10 et $15 \%$ de la surface agricole des exploitations (Gac et al 2010b), constitue un enjeu important. L'ensemble de ces éléments plaide pour raisonner en termes de bilan net, en considérant à la fois les sources de GES et leur compensation par les puits de carbone (Soussana et al 2010).

\section{4 / L'allocation}

Souvent composée de plusieurs ateliers de production, une exploitation agricole commercialise plusieurs produits, ce qui nécessite d'avoir recours à un moyen de répartir les impacts entre ces différents produits. Au regard de la complexité que représente l'allocation, le recours à celle-ci doit être évité dès que l'analyse d'un système de production le permet (ISO 14044), c'est-à-dire par subdivision des procédés de production en sous-procédés et par des collectes séparées de données. C'est pourquoi, une activité agricole doit donc être analysée en prenant soin de ventiler les flux de matières et d'émission par atelier. Deux ateliers composant une exploitation (bovins et cultures ou bovins lait et viande) ou deux composantes d'un atelier (animaux en production et animaux d'élevage) doivent ainsi être analysés séparément (figure 2). Cette situation est rencontrée dans les ateliers laitiers qui produisent à la fois du lait et de la viande et qui possèdent des animaux en production (vaches laitières, animaux à l'engrais) et des ani-

Tableau 3. Proposition de valeurs de flux nets annuels de stockage de carbone additionnel dans le sol, en fonction du mode d'occupation; par hectare, sur l'horizon 0-30 cm, scénario à 20 ans (Gac et al 2010 d'après Arrouays et al 2002, Soussana et al 2010).

\begin{tabular}{|l|l|}
\hline \multicolumn{1}{|c|}{ Type de couvert } & \multicolumn{1}{c|}{$\begin{array}{c}\text { Facteur moyen de } \\
\text { stockage/déstockage }\end{array}$} \\
\hline Prairie de moins de 30 ans & $+500 \mathrm{~kg} \mathrm{C/ha/an}$ \\
\hline Prairie de plus de 30 ans & $+200 \mathrm{~kg} \mathrm{C} / \mathrm{ha} / \mathrm{an}$ \\
\hline Retournement de prairies & $-1000 \mathrm{~kg} \mathrm{C} / \mathrm{ha} /$ an durant 20 ans \\
\hline Culture & $0 \mathrm{~kg} \mathrm{C} / \mathrm{ha} / \mathrm{an}$ \\
\hline \multirow{2}{*}{ Haies } & $+125 \mathrm{~kg} \mathrm{C} / 100$ mètres linéaires de haie \\
\cline { 2 - 2 } & $+100 \mathrm{~kg} \mathrm{C} / \mathrm{ha} / \mathrm{an}$ \\
\hline
\end{tabular}

maux en croissance destinés au renouvellement du troupeau. La problématique est identique pour les systèmes spécialisés de production de viande où sont produits simultanément des femelles réformées, des broutards, des jeunes bovins, etc. Dans la mesure du possible, il convient de distinguer les schémas de production en procédant à l'affectation des émissions à la catégorie animale concernée. Cette affectation des émissions selon les phases de production, consiste à dissocier les processus (figure 2). En atelier laitier, il s'agit d'affecter les émissions associées à la croissance et à l'engraissement des animaux pour la production de viande, et celle des vaches en lactation à la production du lait et du veau.

Néanmoins, lorsqu'une étape du cycle de vie aboutit à plusieurs coproduits (par exemple la viande issue des réformes laitières) et lorsque l'affectation des émissions entre les différentes catégories animales est impossible, le recours à l'allocation est nécessaire afin de répartir l'impact GES entre le lait et la viande issus de ces ateliers laitiers (Cederberg et Stadig 2003, de Vries et De Boer 2010, Kristensen et al 2011). Cinq modes d'allocation principaux entre lait et viande peuvent être identifiés dans le cadre des exploitations laitières :

1/ Allocation au produit majoritaire, où l'ensemble de l'impact de l'atelier laitier est affecté à la production laitière ;

2/ Allocation protéique, basée sur le contenu protéique du lait et de la viande, allocation recommandée par la FAO (2010) ;

3/ Allocation biologique, basée sur l'énergie alimentaire requise pour produire le lait et la viande, allocation recommandée par la FIL (2010) ;

4/ Allocation par extension de système, où les émissions associées à la production de viande bovine (Cederberg et al 2003) ou à une viande mixte bovine et porcine (Nielsen et al 2003) sont soustraites des émissions de l'atelier laitier sur la base d'une empreinte carbone moyenne affectée à la viande ; 
Figure 2. Niveau d'analyse des émissions de gaz à effet de serre par atelier et par phase de production.

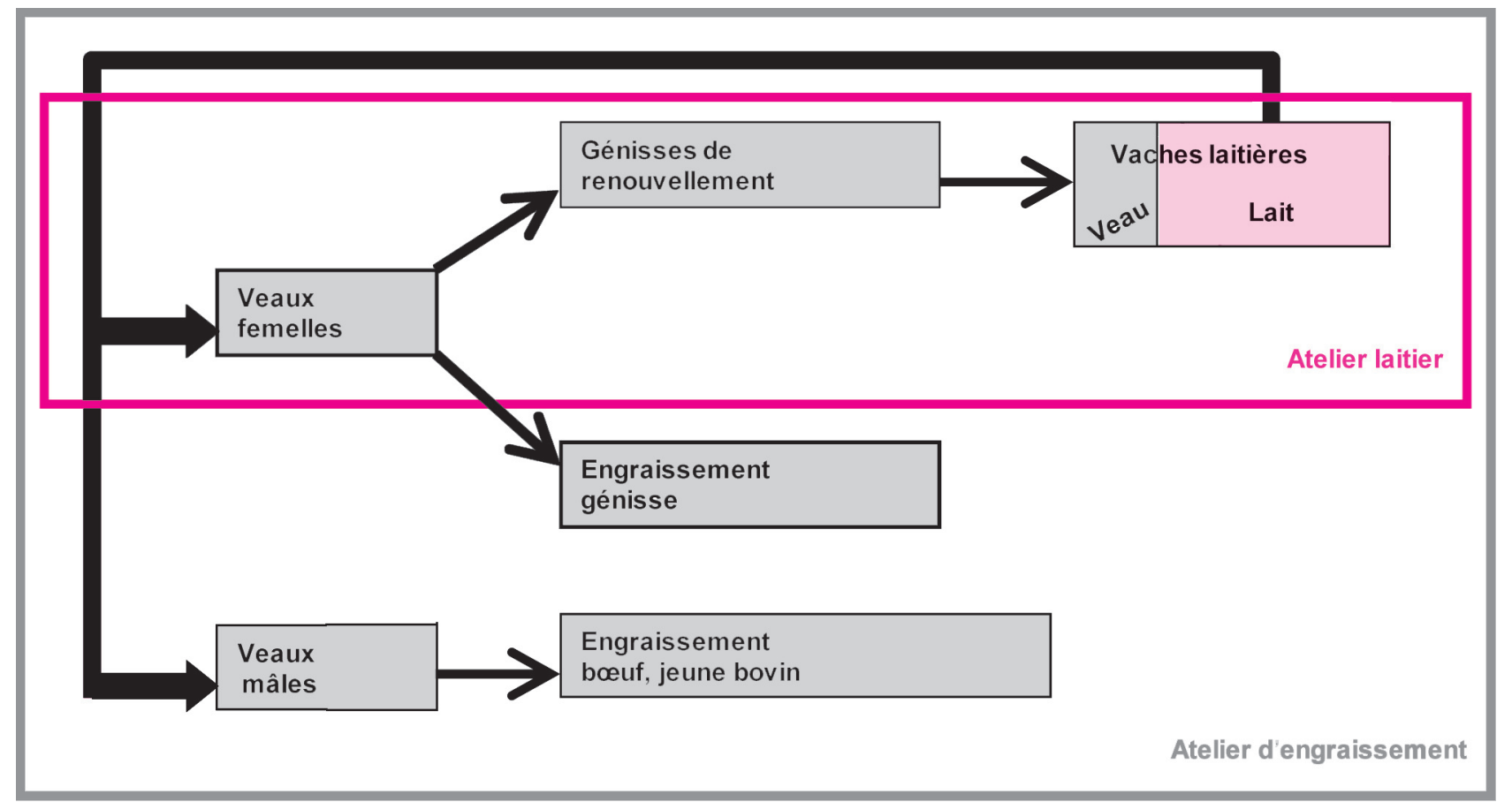

Emissions associées à la production de viande

Emissions associées à la production de lait

5/ Allocation économique, basée sur le revenu économique assuré respectivement par le lait et la viande.

Il est à noter que ces modes de répartition des émissions répondent à trois logiques distinctes. La première est basée sur le fonctionnement du système de production (affectation par phase de production et allocation biologique), la seconde repose sur des clés en lien avec la valeur nutritionnelle du produit final (allocation protéique) et la dernière, sur des éléments de conjoncture (allocation au produit majoritaire, allocation par extension de système, allocation économique). Le choix entre ces clés de répartition des GES doit se faire pour être lisible par les différents acteurs dans le calcul de l'impact mais également pour la mise en œuvre de plans d'action. Les modes d'affectation ou d'allocation basés sur le fonctionnement du système d'exploitation (affectation par phase de production et allocation biologique), revêtent pour cela un intérêt.

Selon le mode d'allocation retenu et le périmètre sur lequel on applique l'allocation choisie, la répartition de l'impact GES entre le lait et la viande est différente (Dollé et al non publié). Sur des ateliers laitiers français, produisant du lait, des veaux et des vaches de réformes, l'impact GES attribué au lait est compris entre 72 et $100 \%$ (tableau 4). Cette distribution de l'impact entre le lait et la viande est sujette à variation en fonction de la conjoncture économique, du ratio lait/viande (Kristensen et al 2011), des performances zootechniques, et a fortiori pour les modes d'allocation protéique et biologique lorsque le périmètre d'analyse est différent. La problématique de l'allocation axée principalement ici sur le troupeau laitier peut dans certains cas concerner les troupeaux allaitants produisant plusieurs types de viande (réforme, bœufs, jeunes bovins, génisses) (Dollé et al 2011). Les principes présentés précédemment (affectation par phase ou allocation) s'appliquent de façon identique à ces systèmes.

Tableau 4. Ratios moyens d'affectation de l'impact GES au lait et à la viande selon différents modes d'allocation (Dollé et al non publié).

\begin{tabular}{|l|c|c|c|c|c|c|}
\hline & $\begin{array}{c}\text { Affectation selon } \\
\text { les phases de } \\
\text { production }\end{array}$ & $\begin{array}{c}\text { Allocation } \\
\text { au produit } \\
\text { majoritaire }\end{array}$ & $\begin{array}{c}\text { Allocation } \\
\text { protéique }\end{array}$ & $\begin{array}{c}\text { Allocation } \\
\text { biologique }\end{array}$ & $\begin{array}{c}\text { Allocation } \\
\text { économique* }\end{array}$ & $\begin{array}{c}\text { Allocation par } \\
\text { extension de } \\
\text { système }\end{array}$ \\
\hline $\begin{array}{l}\text { Emissions } \\
\text { affectées au lait }\end{array}$ & $75 \%$ & $100 \%$ & $88 \%$ & $82 \%$ & $79 \%$ & $72 \%$ \\
\hline $\begin{array}{l}\text { Emissions } \\
\text { affectées à la } \\
\text { viande }\end{array}$ & $25 \%$ & $0 \%$ & $12 \%$ & $18 \%$ & $21 \%$ & $28 \%$ \\
\hline
\end{tabular}

\footnotetext{
* Données RICA (moyennes 2003-2009).

** $1 \mathrm{~kg}$ de viande $=9,5 \mathrm{~kg}$ de $\mathrm{CO}_{2} / \mathrm{kg}$ viande vive.
} 


\section{2 / Evaluation globale à} l'échelle du système d'élevage

Les exploitations d'élevage sont des systèmes complexes avec de multiples interactions entre le cheptel et les surfaces mobilisées. Une évaluation globale à l'échelle du système est nécessaire afin de donner une vision représentative de l'impact environnemental et de pouvoir identifier les leviers d'action visant à réduire les impacts. Cette évaluation globale des émissions de GES ramenées au $\mathrm{kg}$ de produit permet la détermination de l'empreinte carbone brute. La prise en compte du stockage de carbone aboutit à l'appréciation de l'empreinte carbone nette.

\section{1 / Cas des systèmes laitiers}

D’importants travaux ont été réalisés au niveau français afin d'évaluer l'empreinte carbone du lait produit dans les principaux systèmes de production laitière, selon la méthodologie GES'TIM décrite précédemment. Sur un échantillon de 153 exploitations laitières conventionnelles françaises, appartenant à trois systèmes de production différenciés (figure 3), l'empreinte carbone moyenne brute observée est de 1,27 $\mathrm{kg} \mathrm{CO}_{2} / \mathrm{L}$ de lait après application de l'allocation protéique (Dollé et al non publié). La forte contribution du méthane dans l'empreinte carbone du lait (63\%) est liée à la part prépondérante de la fermentation entérique $(69 \%$ des

Figure 3. Empreintes carbone brutes et nettes du lait issu de 3 systèmes laitiers français (Dollé et al non publié).

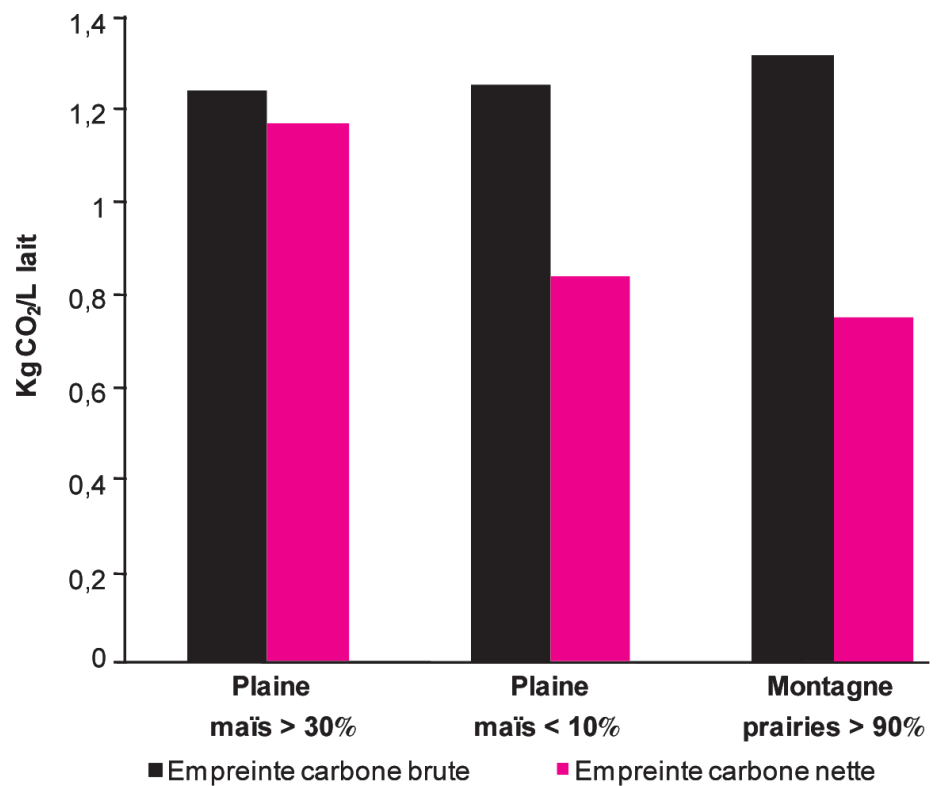

\begin{tabular}{|l|c|c|c|}
\hline & $\begin{array}{c}\text { Plaine } \\
\text { maïs }>\mathbf{3 0 \%}\end{array}$ & $\begin{array}{c}\text { Plaine } \\
\text { maïs }<\mathbf{1 0} \%\end{array}$ & $\begin{array}{c}\text { Montagne } \\
\text { prairies }>\mathbf{9 0} \%\end{array}$ \\
\hline kg lait/vache & 7718 & 5308 & 5860 \\
\hline g concentré/L lait & 209 & 223 & 266 \\
\hline
\end{tabular}

émissions de $\mathrm{CH}_{4}$ ). Les autres émissions se répartissent entre le protoxyde d'azote (17\%), influencé par les émissions au pâturage qui en représentent $41 \%$, et le dioxyde de carbone (20\%), produit par la combustion d'énergies fossiles sur l'exploitation et l'impact des intrants. Ces niveaux d'émission sont cohérents avec la bibliographie qui fait apparaître des empreintes carbone brutes comprises entre 0,8 et $1,5 \mathrm{~kg} \mathrm{CO}_{2}$ par litre (tableau 5). Certaines différen-

Tableau 5. Comparaison de l'empreinte carbone de la production de lait selon différentes sources bibliographiques.

\begin{tabular}{|c|c|c|c|c|c|}
\hline Pays & Sources & Systèmes & $\begin{array}{c}\text { Nombre de } \\
\text { fermes }\end{array}$ & $\begin{array}{c}\text { Emissions brutes } \\
\mathrm{kg} \mathrm{CO} / \mathrm{L}\end{array}$ & $\begin{array}{c}\text { Emissions nettes } \\
\mathrm{kg} \mathrm{CO}_{2} / \mathrm{L}\end{array}$ \\
\hline \multirow{4}{*}{ France } & Gac et al 2010 & Spé. & 196 & $1,2^{(1)}$ & $1,0^{(1)}$ \\
\hline & Dollé et a/ 2009 & Conv. & 4 & $0,8-1,2^{(1)}$ & $0,6-1,0^{(1)}$ \\
\hline & \multirow{2}{*}{$\begin{array}{l}\text { Van der Werf et al } \\
2009\end{array}$} & Conv. & 41 & $1,0^{(2)}$ & - \\
\hline & & $\mathrm{AB}$ & 6 & $1,0^{(2)}$ & - \\
\hline $\begin{array}{l}\text { Nouvelle- } \\
\text { Zélande }\end{array}$ & $\begin{array}{c}\text { Basset-Mens et al } \\
2007\end{array}$ & Conv. & 1 & $0,9^{(2)}$ & - \\
\hline \multirow{3}{*}{ Allemagne } & \multirow{3}{*}{ Haas et al 2001} & Intensif & 6 & $1,3^{(1)}$ & - \\
\hline & & Extensif & 6 & $1,0^{(1)}$ & - \\
\hline & & $\mathrm{AB}$ & 6 & $1,3^{(1)}$ & - \\
\hline Pays-Bas & Vellinga et a/ 2011 & Conv. & 70 & $1,0^{(1)}$ & \\
\hline \multirow{3}{*}{ Suède } & \multirow{2}{*}{ Cederberg et a/ 2000} & Conv. & 1 & $1,1^{(3)}$ & - \\
\hline & & $\mathrm{AB}$ & 1 & $0,9^{(3)}$ & - \\
\hline & Henriksson et a/ 2011 & \multicolumn{2}{|c|}{ Evaluation nationale } & $1,1^{(2)}$ & - \\
\hline \multirow{2}{*}{ Europe } & FAO 2010 & \multicolumn{2}{|c|}{ Ensemble du secteur } & $1,3^{(2)}$ & - \\
\hline & Leip et a/ 2010 & \multicolumn{2}{|c|}{ Lait moyen UE } & $1,4^{(1)}$ & - \\
\hline
\end{tabular}

Spé. : spécialisé ; Conv. : conventionnel ; $A B$ : Agriculture Biologique.

(1) : lait non corrigé ; (2) : lait corrigé sur la matière grasse et protéique ; $(3)$ : lait corrigé sur l'énergie. 
Tableau 6. Efficience environnementale et économique de 64 exploitations appartenant à des systèmes laitiers spécialisés avec plus de $10 \%$ de maïs/ha SFP (Raison et al non publié).

\begin{tabular}{|l|c|c|c|}
\hline & $1 / 4$ Inférieur & Intermédiaire & $1 / 4$ Supérieur \\
\hline Empreinte carbone nette $\left(\mathrm{kg} \mathrm{CO}_{2} / \mathrm{L}\right)$ & 0,93 & 1,14 & 1,30 \\
\hline Nombre d'exploitations & 40 & 57 & 30 \\
\hline Quota $(\mathrm{L})$ & 388712 & 459029 & 464880 \\
\hline Chargement (UGB/SFP) & 1,5 & 1,7 & 1,5 \\
\hline Quantité de concentré par litre de lait $(\mathrm{g} / \mathrm{L})$ & 202 & 222 & 282 \\
\hline Bilan azoté (hors fixation, kg N/ha SAU) & 48 & 91 & 98 \\
\hline Revenu disponible de l'atelier BL (€/UMO) & 35088 & 28795 & 15491 \\
\hline
\end{tabular}

ces sont dues aux systèmes étudiés, à des cas spécifiques avec peu d'exploitations, aux choix méthodologiques (périmètre, règle d'allocation, mode de calcul), ainsi qu'à l'unité fonctionnelle (lait brut ou corrigé sur l'énergie ou la matière grasse et protéique). Néanmoins les résultats sont finalement assez proches au regard de la grande diversité des systèmes mondiaux étudiés, notamment si l'on considère les différences entre les systèmes herbagers avec pâturage intensif de Nouvelle-Zélande et d'Irlande et les systèmes basés sur des stocks comme aux Pays-Bas.

L'analyse des résultats obtenus sur les trois systèmes de production français (figure 3) indique que les émissions brutes de $\mathrm{CO}_{2}$ sont peu différentes d'un système à l'autre. L'impact carbone plus faible des systèmes de plaine à plus forte productivité (7 $718 \mathrm{~kg}$ lait/VL/an) n'est pas significativement différent des résultats obtenus sur les autres systèmes de production. Il est important de noter

Figure 4. Relation entre les émissions de gaz à effet de serre et le niveau de productivité des vaches laitières (Gerber et al 2011).

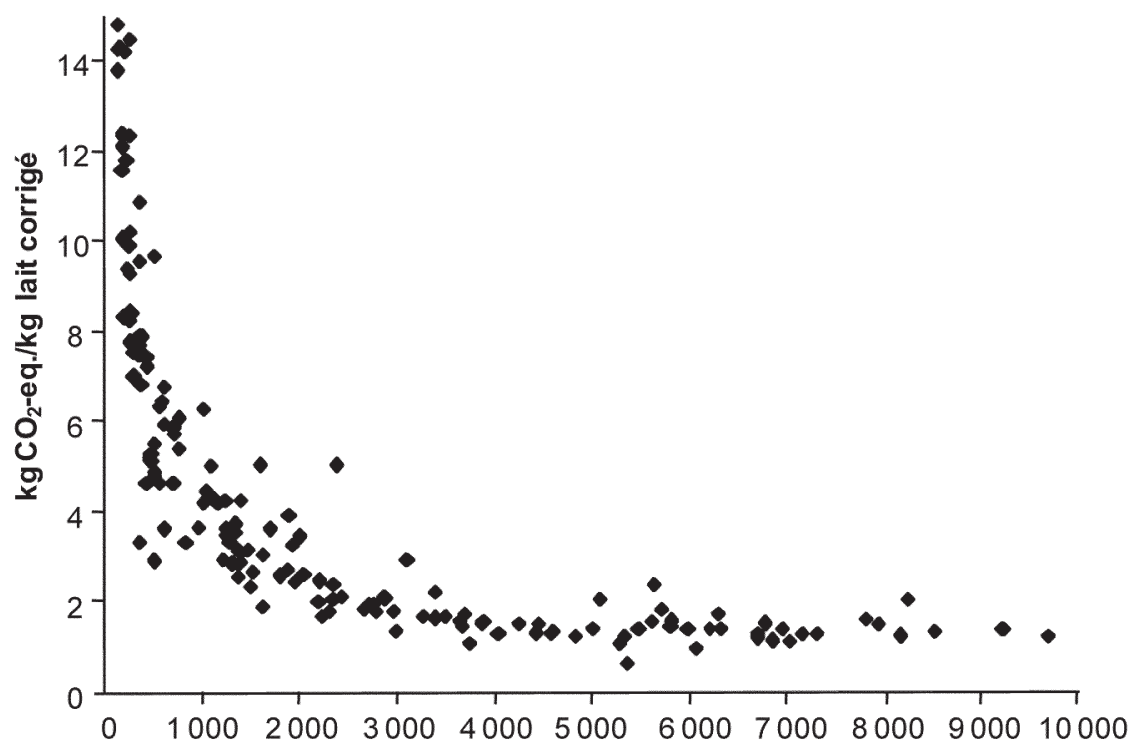

kg lait corrigé/VL/an biologiques (Cederberg et al 2000, Haas et al 2001, Van der Werf et al 2009, Chambaut et al 2011, Kristensen et al 2011). C'est ainsi qu'une émission plus forte de méthane (exprimée en $\mathrm{kg}$ $\mathrm{CO}_{2} / \mathrm{kg}$ de lait) due à une plus faible productivité des systèmes biologiques est généralement compensée par des émissions plus faibles de protoxyde d'azote et de gaz carbonique dues à un moindre recours aux intrants. L'effet du niveau de productivité sur l'empreinte carbone brute exprimée par litre de lait s'observe néanmoins pour des systèmes avec une productivité par vache inférieure à 4000-5000 $\mathrm{kg}$ de lait (Gerber et al 2011) (figure 4), contrairement aux systèmes dont la productivité par vache est supérieure à $5000 \mathrm{~kg}$ de lait (Doreau et Dollé 2011a, Vellinga et al 2011). Par ailleurs, les surfaces en prairie, qui assurent le stockage de carbone dans les sols, représentent une compensation variable des émissions de GES du système comprise entre 6 et $43 \%$ avec une hypothèse de stockage de $500 \mathrm{~kg}$ $\mathrm{C} /$ ha/an (figure 3 ). Le plus fort taux de stockage de carbone obtenu dans les systèmes où la part de prairie est supérieure à $90 \%$, permet une compensation complète du méthane d'origine entérique.

Au sein de la classe typologique «lait spécialisé de plaine», une classification d'exploitations laitières françaises a été réalisée sur la base des résultats de l'empreinte carbone nette (tableau 6). $\mathrm{Au}$ sein de cette population composée de 127 exploitations, on observe une différence de $28 \%$ sur l'empreinte carbone nette du lait entre le quart supérieur et le quart inférieur et une différence de $12 \%$ sur l'empreinte carbone du lait entre le quart supérieur et la classe intermédiaire. Comme observé par Henrikson et al (2011), les variations de l'empreinte carbone sont fortement liées à la gestion du troupeau et aux pratiques culturales. C'est ainsi que les systèmes les plus optimisés, qui possèdent les moindres consommations de concentrés par litre de lait mais également les bilans azotés les plus favorables, 
Tableau 7. Comparaison de l'empreinte carbone de la production de viande selon différentes sources bibliographiques.

\begin{tabular}{|c|c|c|c|c|}
\hline \multirow[t]{2}{*}{ Pays } & \multirow[t]{2}{*}{ Sources } & \multirow[t]{2}{*}{ Systèmes } & $\begin{array}{c}\text { Emissions } \\
\text { brutes }\end{array}$ & $\begin{array}{c}\text { Emissions } \\
\text { nettes }\end{array}$ \\
\hline & & & \multicolumn{2}{|c|}{$\mathrm{kg} \mathrm{CO}_{2} / \mathrm{kg}$ viande vive } \\
\hline \multirow{9}{*}{ France } & \multirow{2}{*}{ Dollé et a/ 2011} & Naisseur & $12,8-14,0$ & $6,5-6,6$ \\
\hline & & Naisseur engr. & $11,3-11,7$ & $7,8-8,5$ \\
\hline & \multirow{3}{*}{ Gac et a/ 2010} & Naisseur & 17,3 & 11,8 \\
\hline & & $\begin{array}{l}\text { Naisseur engr. } \\
\text { Jeune Bovin }\end{array}$ & 14,2 & 11,5 \\
\hline & & $\begin{array}{l}\text { Naisseur engr. } \\
\text { Boeuf }\end{array}$ & 14,2 & 8,9 \\
\hline & Dollé et a/ 2009 & Naisseur engr. & $9,5-12,4$ & $6,4-9,7$ \\
\hline & \multirow{3}{*}{ Veysset et a/ 2011} & Naisseur & $17,2-17,8$ & $13,6-14,6$ \\
\hline & & $\begin{array}{l}\text { Naisseur engr. } \\
\text { Jeune Bovin }\end{array}$ & 14,9 & 12,9 \\
\hline & & $\begin{array}{l}\text { Naisseur engr. } \\
\text { Boeuf }\end{array}$ & 16,9 & 13,6 \\
\hline Irlande & Casey et a/ 2006 & Engr. & $7,6-11,3$ & \\
\hline Suède & Cederberg et al 2002 & Engr. (1) & 6,4 & \\
\hline Europe & Leip et a/ 2010 & $\begin{array}{c}\text { Viande } \\
\text { moyenne UE }\end{array}$ & 22,2 & \\
\hline \multirow[t]{2}{*}{ USA } & \multirow[t]{2}{*}{ Pelletier et a/ 2010} & $\begin{array}{l}\text { Engr. Syst. } \\
\text { pâturant }\end{array}$ & 19,2 & 11,0 \\
\hline & & Engr. Feedlot & 14,8 & 13,0 \\
\hline Brésil & Cederberg et a/ 2011 & & 22 & \\
\hline
\end{tabular}

(1) Viande de jeune bovin laitier.

sont les plus performants sur le plan environnemental. Enfin, cette analyse permet au regard des résultats économiques et environnementaux de mettre en évidence le lien entre efficiences économique et environnementale. Les défauts d'optimisation technique qui se traduisent par une empreinte carbone plus élevée, génèrent des résultats économiques plus faibles (tableau 6).

\section{2 / Cas des systèmes produc- teurs de viande}

Les travaux conduits en France sur l'évaluation de la production de viande issue des troupeaux allaitants conventionnels (Dollé et al 2009, Gac et al 2010c, Dollé et al 2011, Veysset et al 2011) font état d'une empreinte carbone brute comprise entre 9,5 et $17,8 \mathrm{~kg}$ $\mathrm{CO}_{2} / \mathrm{kg}$ viande vive (vv) (tableau 7), sans distinction du type de viande produit (vache de réforme, jeune bovin, bœufs...). La contribution des trois principaux GES met l'accent sur la part prépondérante du méthane $(71 \%)$. La fermentation entérique représente $52 \%$ des émissions totales soit $73 \%$ du méthane total émis. Les émissions en bâtimentstockage pèsent pour $21 \%$. Les émissions de $\mathrm{N}_{2} \mathrm{O}$ représentent $17 \%$ de l'empreinte totale et sont essentiellement liées au pâturage (42\%). Les émissions de $\mathrm{CO}_{2}$, dues à la consommation d'énergies directes et essentiellement aux intrants, sont de $12 \%$. Ces empreintes carbone brutes des systèmes bovins vian- de français évaluées avec deux méthodologies distinctes, GES'TIM pour Gac et al (2010a) et Dollé et al (2011) et PLANETE-OPT'INRA pour Veysset et al (2011), sont inférieures à celle qui est évaluée pour la viande bovine au niveau européen et qui atteint $22,2 \mathrm{~kg}$ de $\mathrm{CO}_{2} / \mathrm{kg}$ vv (Leip et al 2010). Cette moyenne cache cependant des disparités importantes. L'Autriche $\left(14,2 \mathrm{~kg} \mathrm{CO}_{2} / \mathrm{kg}\right.$ vv) et les Pays-Bas $(17,4 \mathrm{~kg} \mathrm{CO} / \mathrm{kg}$ vv) possèdent l'empreinte carbone brute la plus faible comparativement à Chypre $(44,1 \mathrm{~kg} \mathrm{CO} / \mathrm{kg} \mathrm{vv})$ et à la Lettonie $(41,8 \mathrm{~kg} \mathrm{CO} / \mathrm{kg} \mathrm{vv})$ où la productivité plus faible des systèmes de production est associée à un changement important d'occupation des terres. C'est ainsi que les régimes équilibrés couplés à des durées d'engraissement optimisées (Benchaar et al 2001, Lovett et al 2005, Casey et Holden 2006, Dollé et al 2011) permettent de réduire l'impact carbone ramené au $\mathrm{kg}$ de viande produit. L'incidence de la productivité est également mise en évidence par Pelletier et al (2010), l'empreinte carbone brute des bovins engraissés aux Etats-Unis sur des systèmes pâturant non optimisés étant supérieure à celles des bovins engraissés en feedlot (19,2 $\mathrm{kg} \mathrm{CO}_{2} / \mathrm{kg}$ vv vs $14,8 \mathrm{~kg}$ $\mathrm{CO}_{2} / \mathrm{kg} \mathrm{vv}$ ) du fait d'un gain de poids quotidien plus faible $(0,6 \mathrm{~kg}$ vv/jour $v s$ $1,4 \mathrm{~kg} \mathrm{vv} /$ jour).

Cette empreinte carbone de la viande des troupeaux allaitants intègre la phase d'élevage des broutards ainsi que la phase d'engraissement. Parmi les émissions de ces systèmes de production, les vaches nourrices représentent la plus grosse part. Les systèmes «naisseurs», où les vaches représentent l'essentiel des effectifs présents et où la quantité de viande valorisée est plus faible, possèdent ainsi une empreinte carbone plus forte comparativement aux élevages naisseurs-engraisseurs. Sur la seule phase d'engraissement, Doreau et al (2011b) ont ainsi mis en évidence une empreinte carbone comprise entre 3,7 et $4,7 \mathrm{~kg} \mathrm{CO} / 2 \mathrm{~kg}$ de gain de vv selon la part de fourrages et de concentrés dans la ration; comparativement aux systèmes naisseurs qui affichent une empreinte carbone brute comprise entre 12,8 et $17,8 \mathrm{~kg} \mathrm{CO} / \mathrm{kg}$ vv (Gac et al 2010c, Dollé et al 2011, Veysset et al 2011). C'est pourquoi, les émissions de GES liées à l'élevage des broutards représentent de 75 (Dollé et al 2009) à $80 \%$ (Phetteplace et al 2001) de l'empreinte finale. La phase d'allaitement en lien avec la conduite des mères est la source essentielle de GES sur l'ensemble du cycle de vie de l'animal et influe ainsi considérablement l'empreinte carbone du produit final.

La détermination de l'empreinte carbone nette considérant le stockage de carbone a été encore peu étudiée pour les systèmes viande. Les travaux de Dollé et al (2009), Gac et al (2010b), Dollé et al (2011) mettent en évidence une compensation des émissions de 
GES comprise entre 24 et $53 \%$ pour un stockage de carbone des prairies fixé à $500 \mathrm{~kg} \mathrm{C} / \mathrm{ha} / \mathrm{an}$. Sur la base d'un stockage carbone par les prairies de 350 $\mathrm{kg} / \mathrm{ha} / \mathrm{an}$, Veysset et al (2011) font état d'une compensation comprise entre 13,3 et $21,2 \%$ selon la part de prairies valorisées dans le système. C'est ainsi que des systèmes plus intensifs (part importante de concentrés, systèmes faiblement pâturant, GMQ élevé), qui possèdent une empreinte carbone brute exprimée en $\mathrm{kg} \mathrm{CO}_{2} / \mathrm{kg}$ vv plus faible, sont associés à une faible compensation carbone et ainsi aboutissent à une empreinte carbone nette identique voire supérieure aux autres systèmes (Dollé $e t$ al 2011, Veysset et al 2011). Pelletier et al (2010) qui ont utilisé les valeurs proposées par Phetteplace et al (2001), mettent en avant une compensation carbone de $42 \%$ pour les systèmes pâturant contre $12 \%$ pour les bovins engraissés en feedlot. Les systèmes pâturant optimisés possèderaient ainsi une empreinte carbone nette de $15 \%$ inférieure aux systèmes intensifs (Pelletier et al 2010).

La conversion du système de production en agriculture biologique (Chambaut et al 2011, Veysset et al 2011) se traduit par une empreinte carbone nette plus faible, notamment du fait de l'importance des prairies dans le système biologique. En faisant abstraction du stockage de carbone, Casey et Holden (2006) font état d'un niveau d'émission exprimé par $\mathrm{kg}$ de viande vive de $14 \%$ inférieur pour les systèmes biologiques, alors que Haas et al (2001) ne trouvent pas de différence significative entre les deux modes de production.

\section{3 / Cas de la viande produite dans les systèmes laitiers}

Même si le principal produit des systèmes laitiers est le lait, leur production de viande représente une part non négligeable évaluée à $40 \%$ de la viande bovine produite en France. Sur un atelier laitier, où sont élevés conjointement les animaux de renouvellement et en production, il convient de répartir les émissions entre les deux produits valorisés lait et viande. Plusieurs modes de répartition des émissions de GES décrits précédemment ont été appliqués sur des systèmes laitiers (Dollé et al non publié) afin de déterminer l'empreinte carbone nette respective du lait et de la viande issue des animaux de réforme. L'incidence des différents modes d'allocation sur l'empreinte carbone est très forte (figure 5). L'affectation des émissions par phase de production, qui consiste à attribuer les émissions des animaux de renouvellement à la viande et les émissions de la phase de lactation et de tarissement au lait et au veau, conduit à une empreinte carbone du lait de $0,83 \mathrm{~kg} \mathrm{CO} / \mathrm{kg}$ et à une empreinte carbone de la viande de réforme laitière de $8,7 \mathrm{~kg} \mathrm{CO} / \mathrm{kg}$ vv. Allouer les émissions de GES selon les besoins alimentaires des animaux (FIL 2010) pour la production de lait et de viande (allocation biologique), se traduit par une empreinte carbone du lait de $0,9 \mathrm{~kg}$ $\mathrm{CO}_{2} / \mathrm{kg}$ et de la viande de $6,4 \mathrm{~kg}$ $\mathrm{CO}_{2} / \mathrm{kg}$ vv. Ces deux modes de répartition (biologique et affectation par phase de production) en lien avec le système de production, conduisent à une empreinte carbone plus proche de l'allocation par extension de système, où l'empreinte de la viande correspond à la moyenne des viandes des systèmes allaitants spécialisés. L'application de l'allocation protéique recommandée par la FAO (2010), aboutit à une empreinte carbone de la viande plus faible $(4,4 \mathrm{~kg}$ $\mathrm{CO}_{2} / \mathrm{kg} \mathrm{vv}$ ). Enfin, une allocation au produit majoritaire où l'ensemble des émissions est affectée au lait produit se traduit par une empreinte carbone du lait la plus forte $\left(1,1 \mathrm{~kg} \mathrm{CO}_{2} / \mathrm{kg}\right.$ lait). A l'exception des résultats obtenus avec l'allocation économique, où le ratio lait/viande dépend fortement de la conjoncture économique et des recettes retenues (avec ou sans aides), les empreintes calculées sont en concordance avec les résultats de Kristensen (2011) qui a réalisé la même évaluation.

Par ailleurs, les émissions de GES de la vache laitière en lactation étant pour une part importante affectées au lait, le veau issu des troupeaux laitiers possède une empreinte carbone plus faible que celui produit en système allaitant. Une évaluation conduite sur des systèmes français spécialisés dans l'engraissement des jeunes bovins issus de troupeaux laitiers fait état d'une empreinte carbone brute de $4,5 \mathrm{~kg} \mathrm{CO} / \mathrm{kg}$ vv. Ces résultats corroborent ceux obtenus par Cederberg et Darelius (2002) où l'empreinte carbone est de $6,5 \mathrm{~kg} \mathrm{CO}_{2} / \mathrm{kg} \mathrm{vv}$.

Figure 5. Empreintes carbone nettes du lait et de la viande issus des ateliers laitiers selon différents modes d'allocation (Dollé et al non publié).

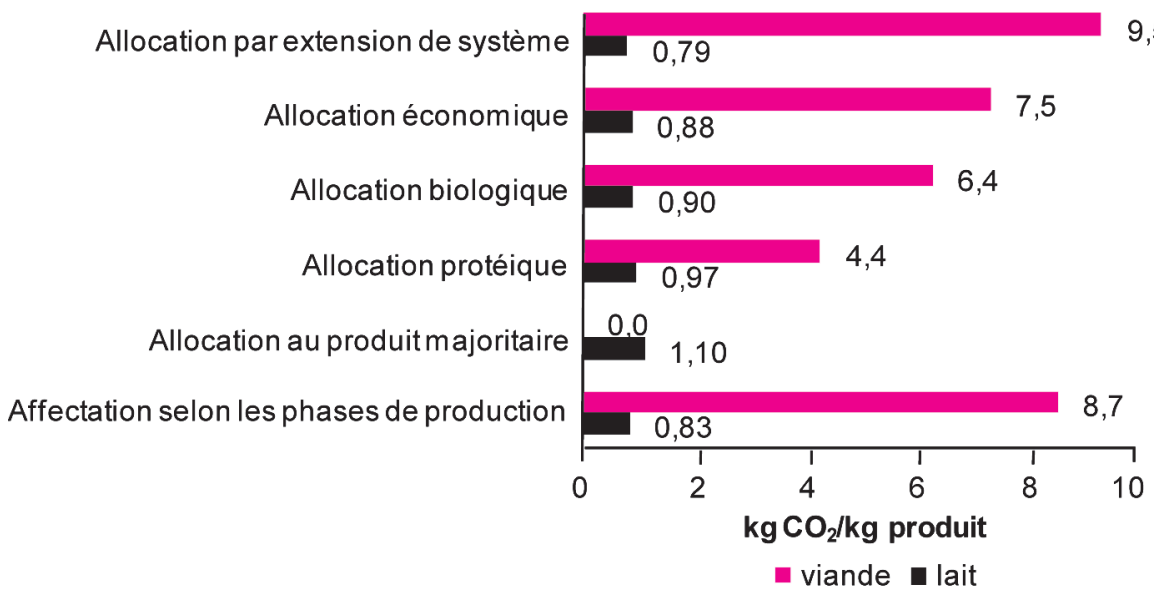

Il est à noter que dans tous les cas, les systèmes spécialisés lait et mixtes lait/viande conduisent à une empreinte carbone nette de la viande inférieure aux ateliers viande spécialisés.

\section{3 / Les leviers d'action}

Pour satisfaire les objectifs nationaux, européens et internationaux, des recommandations techniques sont mises en avant (Metz et al 2007). Les différences observées entre systèmes de production et entre exploitations d'un même système (Henriksson et al 2011) mettent en évidence les potentialités en matière de réduction de l'empreinte carbone des produits lait et viande bovine. Des stratégies existent pour réduire les émissions de méthane, de protoxyde d'azote et de gaz carbonique. Celles-ci doivent être évaluées et testées à l'échelle du système et pour les trois GES concernés de manière à éviter tout transfert de pollution d'un gaz à un autre ou d'un compartiment à un autre.

\section{1 / Alimentation des animaux}

L'alimentation est un levier d'action pour réduire les émissions de méthane entérique et les rejets azotés des animaux. L'augmentation de la part de concentrés dans la ration, qui remplace les glucides des parois végétales des fourrages par de l'amidon et des sucres est ainsi souvent citée comme étant un moyen de réduire les émissions de méthane entérique (Martin et al 2010, Doreau et al 2011a). Toutefois, Hindrichsen et al (2006) mettent en évitions animales supérieures pour des vaches laitières alimentées avec du fourrage et des concentrés comparativement à un régime composé de fourrage seul. Au-delà de l'effet sur le méthane, cette option technique, qui consiste à dence des émissions liées aux déjec- 
Tableau 8. Effets potentiels des principaux leviers d'action sur les émissions de GES.

\begin{tabular}{|c|c|c|c|c|}
\hline Leviers d'action & Méthane & $\begin{array}{l}\text { Protoxyde } \\
\text { d'azote }\end{array}$ & $\begin{array}{c}\text { Gaz } \\
\text { carbonique }\end{array}$ & $\begin{array}{l}\text { Effet potentiel sur la } \\
\text { réduction de } \\
\text { l'empreinte carbone } \\
\text { nette des produits }\end{array}$ \\
\hline \multicolumn{5}{|l|}{ Alimentation des animaux } \\
\hline Augmentation de la part de concentrés & $\downarrow$ & - & $\uparrow$ & 0 à $5 \%$ \\
\hline Apports de lipides & $\downarrow$ & - & - & 3 à $7 \%$ \\
\hline Additifs alimentaires & $\downarrow$ & $\downarrow$ & - & $?$ \\
\hline $\begin{array}{l}\text { Optimisation de la teneur en azote de la } \\
\text { ration }\end{array}$ & - & $\downarrow$ & $\downarrow$ & 2 à $5 \%$ \\
\hline $\begin{array}{l}\text { Remplacement du tourteau de soja par du } \\
\text { tourteau de colza }\end{array}$ & - & - & $\downarrow$ & 3 à $7 \%$ \\
\hline Autonomie protéique & - & - & $\downarrow$ & 2 à $5 \%$ \\
\hline \multicolumn{5}{|l|}{ Productivité et gestion du troupeau } \\
\hline Productivité & $\downarrow$ & $\downarrow$ & $\downarrow \uparrow$ & -5 à $10 \%$ \\
\hline Renouvellement & $\downarrow$ & $\downarrow$ & $\downarrow$ & 0 à $5 \%$ \\
\hline Optimisation sanitaire & $\downarrow$ & $\downarrow$ & $\downarrow$ & 2 à $5 \%$ \\
\hline Amélioration génétique & $\downarrow$ & $\downarrow$ & $\downarrow$ & 2 à $10 \%$ \\
\hline \multicolumn{5}{|l|}{ Fertilisation azotée } \\
\hline Réduction des apports $\mathrm{N}$ & - & $\downarrow$ & $\downarrow$ & 2 à $5 \%$ \\
\hline Introduction de légumineuses & - & $\downarrow$ & $\downarrow$ & 2 à $5 \%$ \\
\hline Inhibiteur de nitrification & - & $\downarrow$ & $\downarrow$ & 0 à $5 \%$ \\
\hline \multicolumn{5}{|l|}{ Gestion des déjections } \\
\hline Optimisation du pâturage & $\downarrow$ & $\downarrow$ & - & 3 à $5 \%$ \\
\hline Valorisation optimale des déjections & - & $\downarrow$ & $\downarrow$ & 3 à $5 \%$ \\
\hline Méthanisation & $\downarrow$ & - & $\downarrow$ & 5 à $7 \%$ \\
\hline \multicolumn{5}{|l|}{ Réduction des consommations d'énergie } \\
\hline Electricité & - & - & $\downarrow$ & $<1 \%$ \\
\hline Fioul & - & - & $\downarrow$ & 1 à $2 \%$ \\
\hline \multicolumn{5}{|l|}{ Stockage de carbone } \\
\hline Augmenter la part de PP & - & - & $\downarrow$ & 3 à $10 \%$ \\
\hline Implanter des haies & - & - & $\downarrow$ & 3 à $10 \%$ \\
\hline
\end{tabular}

augmenter la part de concentré dans la ration, peut entraîner un accroissement des coûts de production et met les ruminants en concurrence avec l'Homme pour l'accès aux ressources alimentaires. Par ailleurs, la production des concentrés a elle-même un impact GES qui rend cette solution moins attrayante que ne le laisse présager une analyse rapide. C'est ainsi qu'il est constaté pour des exploitations laitières françaises (tableau 6) une surconsommation de $28 \%$ de concentré entre les élevages non optimisés possédant l'empreinte carbone la plus élevée et les élevages optimisés à l'empreinte carbone plus faible (Raison et al non publié). Sur la phase d'engraissement seule, ce gain sur le méthane entérique permis par les régimes à très haute teneur en concentré $(>70 \%)$ reste acquis à l'échelle du système, malgré des émissions plus importantes de $\mathrm{N}_{2} \mathrm{O}$ et de $\mathrm{CO}_{2}$ (Doreau et al 2011b). Un autre levier pour réduire les émissions de méthane entérique concerne l'enrichissement de la ration en lipides insaturés, qui permet une diminution moyenne de 3,8\% des émissions de méthane pour $1 \%$ de lipides supplémentaire dans la ration (Martin et al 2010). L'effet sur le méthane entérique est néanmoins très dépendant de la nature des lipides employés (acides laurique, myristique, linoléique) (Martin et al 2010). Certains auteurs (Woodward et al 2006) remettent par ailleurs en cause l'effet à long terme des lipides sur les émissions de méthane, mais des données récentes obtenues avec de la graine de lin mettent bien en évidence un effet positif à long terme sur les émissions entériques (Martin et al 2011). Les additifs alimentaires (ionophores, acides organiques) pourraient constituer une autre voie de réduction des émissions de méthane entérique, cependant leur développement est encore sujet à de nombreuses interrogations (Doreau et al 2011a). Certaines substances sont interdites en Europe (antibiotiques ionophores), d'autres sont d'origine chimique et très coûteuses (acides organiques), d'autres enfin n'ont pas fait preuve d'un effet durable dans le temps (tannins, saponines).

Concernant les rejets azotés des animaux, une évaluation a montré qu'une diminution du contenu azoté du régime semble être une voie moins efficace que des actions sur la gestion des engrais (Henriksson et al 2011). Cependant, la réduction des pertes fécales et particulièrement urinaires représente une piste intéressante (Kebreab et al 2001) qui permet de limiter les émissions de $\mathrm{N}_{2} \mathrm{O}$ liées à la gestion des déjections animales mais également les émissions de $\mathrm{CO}_{2}$ associées à la production des 
intrants. Une bonne adéquation des apports aux besoins constitue donc une voie intéressante et simple pour réduire la consommation de concentrés et les pertes azotées. Il semble même possible dans certains cas de réduire les apports d'azote dégradable un peu en dessous des recommandations pour accroître l'efficience d'utilisation de l'azote et diminuer encore les rejets sous forme d'urée sans trop pénaliser les performances. Cette marge de manœuvre peut être utilisée chez des ruminants par ailleurs bien alimentés en acides aminés (Vérité et al 1997). Un déficit de 5\% du niveau d'apport d'azote dégradable par rapport aux recommandations n'aura ainsi pas de conséquences sur les performances de production mais permettra de réduire les rejets d'environ $10 \mathrm{~kg}$ de $\mathrm{N}$ par vache sur une lactation. Une réduction plus importante, conduira à diminuer fortement les performances.

Une autre piste en alimentation animale concerne l'achat d'aliments à faible impact carbone en lien avec les pratiques culturales ou le changement d'affectation des sols (colza vs soja). Enfin la recherche d'une autonomie alimentaire de l'exploitation constitue également un levier de réduction des émissions de GES. Cela concerne en particulier la recherche d'une autonomie en protéines sur les exploitations par l'utilisation d'herbe et de légumineuses. Chacun des leviers ici évoqués pour l'alimentation, permet une réduction des émissions de GES comprise entre 0 et $7 \%$ selon les cas (tableau 8 ). D'importants travaux de recherche sont néanmoins encore nécessaires pour préciser l'intérêt environnemental et technico-économique de ces leviers.

\section{2 / Productivité et gestion du troupeau}

L'augmentation de la productivité laitière est fréquemment suggérée pour réduire l'impact méthane entérique ramené au kg de produit et pourrait permettre de réduire l'empreinte carbone de $0,03 \mathrm{~kg} \mathrm{CO} / \mathrm{kg}$ lait (Schils et al 2005) à $0,26 \mathrm{~kg} \mathrm{CO}_{2} / \mathrm{kg}$ lait (Vellinga et al 2011). Cette tendance est toutefois remise en cause selon le système de production étudié (Gerber et al 2011) et le niveau de productivité compte tenu de l'empreinte carbone des intrants, des émissions gazeuses supérieures en bâtiment d'élevage comparativement à la période de pâturage, et d'une moindre compensation carbone par les prairies. L'évaluation de l'empreinte carbone conduite sur l'échantillon de 153 exploitations laitières françaises (Dollé et al non publié) montre une faible tendance à la diminution de l'empreinte carbone brute avec l'augmentation de productivité (figure 6a). Sur le même
Figure 6. Empreintes carbone brutes (6a) et nettes (6b) du lait selon la productivité par vache dans 153 exploitations laitières (Dollé et al non publié).
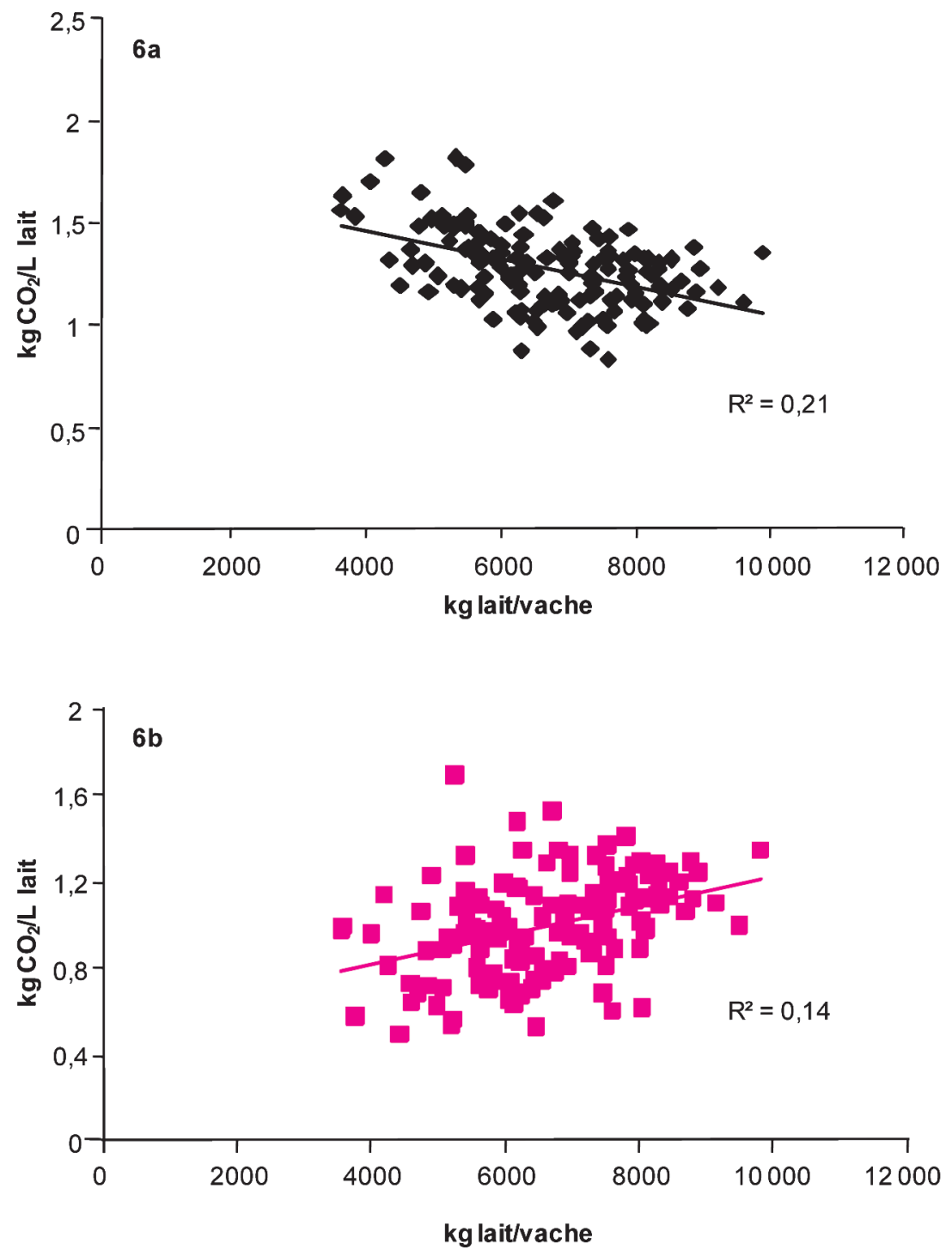

échantillon, la prise en compte du stockage de carbone dans le calcul de l'empreinte nette, met en évidence l'impact négatif que pourrait avoir une augmentation de la productivité au détriment des surfaces en prairies (figure 6b).

Par ailleurs, Lovett et al (2006) précisent que le gain obtenu sur le méthane entérique de vaches à haut potentiel peut être perdu si l'on regarde l'ensemble de la carrière de la vache et le nombre de femelles de renouvellement nécessaires. Ainsi, des situations d'élevage avec un nombre de lactations et des performances de reproduction généralement plus faibles, des animaux plus sensibles aux risques sanitaires, sont associées à un taux de renouvellement plus fort et donc à une part d'animaux ne produisant pas de lait plus élevée. La part des émissions de GES du troupeau de renouvellement représentant 25 à $35 \%$ des émissions de l'atelier laitier, toute action réduisant la proportion d'a- nimaux en croissance et en entretien par rapport aux animaux en production doit être étudiée. Une diminution du taux de renouvellement comprise entre 5 et $9 \%$ pourrait ainsi permettre une réduction de l'empreinte carbone de 20 à $39 \mathrm{~g}$ $\mathrm{CO}_{2} / \mathrm{kg}$ de lait (Vellinga et al 2011) à l'échelle d'une exploitation. Ces observations méritent toutefois d'être relativisées car les gains mis en évidence sur des ateliers laitiers sont dépendants du mode d'affectation ou d'allocation de l'impact entre le lait et la viande. C'est ainsi que toute action sur le renouvellement d'un troupeau laitier peut se traduire par un effet sur l'empreinte carbone du lait pour des modes d'allocation protéique, économique, etc. mais sera sans effet sur l'impact final du lait si l'on adopte une affectation par phase de gique. C'est ainsi qu'à l'échelle globale de l'élevage bovin producteur de lait et de viande, ces pistes de réduction de l'empreinte carbone du lait par augmenproduction ou une allocation biolo- 
tation de la productivité ou par réduction du taux de renouvellement de l'atelier laitier seraient sans effet sur les émissions totales de GES. Pour satisfaire une même demande en lait et viande, tout effort centré sur l'augmentation de productivité laitière et le nombre de lactations serait en défaveur de la «viande coproduit» du lait et nécessiterait une production plus importante de viande issue des ateliers spécialisés dont l'empreinte carbone est plus élevée (Zehetmeier et al 2011). La situation est différente pour les bovins viande en phase d'engraissement. Sous réserve que cette augmentation de productivité ne soit pas associée à un recours important d'intrants, celle-ci présente un intérêt dans la mesure où un gain de poids moyen quotidien supérieur permet de réduire sensiblement la durée d'engraissement et les émissions associées.

Enfin, une meilleure gestion du troupeau (réforme des animaux peu productifs, gestion sanitaire efficace, capacité d'adaptation aux modifications de l'environnement...) représente un potentiel de réduction des émissions de GES de l'élevage bovin compris entre 2 et $5 \%$ (Cruickshank et al 2009). D'autres pratiques qui touchent davantage à la gestion du troupeau allaitant, comme le choix de la date de mise bas selon la date de pousse maximale de l'herbe pour optimiser le pâturage, doivent encore être évaluées.

L'amélioration génétique représente également une opportunité pour réduire les émissions de GES. En axant la sélection sur l'amélioration de l'efficacité alimentaire, Alford et al (2006) estiment le potentiel de réduction des émissions de méthane à $3,1 \%$ sur une durée de 25 ans. De même, l'efficience de production obtenue par une meilleure fertilité, une valorisation optimale de la ration, une baisse de la dépense d'entretien et une amélioration de la santé des animaux (Hegarty 2004) constitue une voie importante de progrès. Sur la base des données bibliographiques et des premières évaluations effectuées sur les systèmes français, toute action agissant sur la productivité et la gestion du troupeau, peut selon les cas permettre une réduction de l'empreinte carbone comprise entre 0 et $10 \%$ (tableau 8 ).

\section{3 / Fertilisation azotée}

De Klein et Eckard (2008) suggèrent que les émissions de protoxyde d'azote puissent être réduites de $90 \%$ avec une gestion adéquate de l'azote. Même si cette valeur semble surestimée, il peut être acté que les émissions de protoxyde d'azote peuvent être atténuées en grande partie, et une grande variété de solutions techniques peut être explorée (Luo et al 2010). L'indicateur majeur associé à la gestion de l'azote est l'excédent d'azote à l'échelle de l'exploitation, solde entre les apports d'azote (azote des intrants) et l'azote mobilisé dans les produits du système. Cet excédent représente un risque de lessivage, de perte par volatilisation ammoniacale et par émission de protoxyde d'azote. En matière de fertilisation, une bonne gestion et une valorisation agronomique optimale des déjections animales permettent de réduire fortement l'achat d'engrais minéraux. Le bilan entrée/sortie de l'azote montre que $80 \%$ des fermes françaises d'herbivores ont un excès d'azote inférieur à $90 \mathrm{~kg} / \mathrm{ha}$ (Le Gall et al 2009) en adéquation avec de faibles pertes de nitrates. Des efforts importants ont été faits durant les dernières années (ouvrages de stockage, plan de fertilisation...) pour réduire l'utilisation d'engrais minéraux ; cependant les efforts doivent se poursuivre. Au-delà de la réduction des émissions de protoxyde d'azote, toute optimisation de la fertilisation azotée réduit le recours aux achats d'engrais de synthèse et ainsi aux émissions de gaz carbonique liées à leur fabrication et à leur transport, soit entre 5,3 et $6,1 \mathrm{~kg}$ $\mathrm{CO}_{2} / \mathrm{kg}$ d'azote.

Le recours aux légumineuses constitue également une piste de réduction des émissions de protoxyde d'azote du fait de la fixation symbiotique et de la réduction d'émissions de protoxyde liées aux conditions aérobies. Le GIEC considère ainsi que la fixation symbiotique n'émet pas de GES (IPCC 2006). Corré et al (2002) font état d'émissions de $\mathrm{N}_{2} \mathrm{O}$ mesurées sur les prairies d'associations plus faibles que celles mesurées sur les prairies de graminées $(0,2 v s$ $1,3 \%$ N). Ledgard et al (2009) mettent en évidence qu'un lait produit sur une prairie fertilisée avec $200 \mathrm{~kg}$ d'azote se traduit par une empreinte carbone supérieure à $15 \%$ à celle d'un lait produit sur une prairie non fertilisée et contenant des légumineuses. Les légumineuses sont ainsi un des leviers efficaces pour réduire le recours aux engrais de synthèse. Les principales difficultés portent sur la pérennité du trèfle blanc dans les prairies et le sursemis de trèfle blanc dans les prairies installées.

Les inhibiteurs de nitrification, qui visent à ralentir la production d'azote nitrique issu de la transformation de l'azote ammoniacal des pissats et des déjections, peuvent également réduire les pertes par lessivage et les émissions de $\mathrm{N}_{2} \mathrm{O}$. Peu utilisés en France, mais très étudiés en Nouvelle-Zélande (Monagham et al 2007), ces produits sont appliqués par épandage sur les prairies ou mélangés aux produits organiques avant épandage. Certains travaux mettent en avant une réduction des émissions de GES de 50 à $68 \%$ issus des urines au pâturage grâce à l'application d'inhibiteurs de nitrification (Monaghan et al 2007 et 2009, Di et Cameron 2010) soit jusqu'à 5\% de l'empreinte carbone du produit final. D'autres travaux rapportent des résultats beaucoup moins positifs, voire pas d'effets des inhibiteurs de nitrification (Mc Donald 2010). Les résultats variant selon les sites expérimentaux, les conditions d'apport (date et dose) et les supports, des travaux vont être conduits afin d'apprécier l'efficacité en France de ces inhibiteurs de nitrification. Les trois pistes évoquées ici peuvent permettre une réduction des émissions de GES et de l'empreinte carbone comprise entre 0 et $5 \%$ (tableau 8).

\section{4 / La gestion des déjections}

La gestion des déjections couvre à la fois les déjections produites au pâturage et celles générées en bâtiment. Au pâturage, les déjections sont beaucoup moins émettrices que celles produites en bâtiment puis stockées (IPCC 2006). Une piste majeure dans la gestion des effluents peut donc consister à réduire les quantités stockées en augmentant le pâturage. De gros investissements ont été contractés ces dernières années afin d'optimiser la gestion des déjections générées en bâtiment sur le site d'exploitation (collecte, ouvrages de stockage de longue durée) et leur valorisation agronomique. Cette meilleure gestion se traduit par des apports aux périodes appropriées, au plus près des besoins de plantes, ce qui permet de réduire les apports d'azote minéral. La gestion des déjections sur site peut être complétée par la digestion anaérobie des déjections animales afin d'éviter les émissions de méthane au stockage. Le biogaz, mélange de $\mathrm{CH}_{4}$ et de $\mathrm{CO}_{2}$, est valorisé sous forme énergétique. En système laitier, l'installation d'une unité de méthanisation peut permettre un abattement de l'empreinte carbone comprise entre 5 et $7 \%$ (Dollé et al non publié). Toutefois la mise en place d'un tel dispositif, qui est conditionnée par un certain nombre de paramètres tels que la production constante de déjections, la disponibilité en co-substrats, la valorisation de la chaleur, ne pourra toutefois concerner qu'un nombre limité d'installations.

Le type de déjections produites, lisier ou fumier, a également une incidence sur les émissions de méthane et de protoxyde d'azote en bâtiment et au stockage. Les conditions de production et de stockage des fumiers (sur plateforme ou sous les animaux) agissent notamment sur les niveaux d'émission 
à travers le degré d'anaérobiose. Peu de références bibliographiques étant disponibles sur les émissions associées à la gestion des déjections sous forme de fumier, des travaux sont en cours sur les différents modes de logements rencontrés en France (aire raclée et litière accumulée) afin de préciser les mécanismes et les niveaux d'émission.

\section{5 / Réduction des consomma- tions d'énergie}

Les émissions de gaz carbonique «énergétiques» émanent des consommations de fioul et d'électricité. Des solutions techniques très intéressantes pour réduire les consommations électriques (pré-refroidisseur de lait, récupérateur de chaleur, solaire thermique) se traduisent au final par un gain sur l'empreinte carbone inférieur à $1 \%$ compte tenu de l'origine nucléaire de l'électricité produite en France (Dollé et al non publié). Les actions visant à réduire les consommations de fioul peuvent concerner à la fois une évolution du système de production et des pratiques. Le pâturage constitue pour cela une des pistes de réduction des consommations de fioul pour la culture, la récolte, la distribution des fourrages et l'épandage des déjections. Par ailleurs, les modifications du comportement et des pratiques (conduite économe, réglage des tracteurs, réduction du transport, simplification des pratiques culturales...) représentent également un intérêt non négligeable pouvant permettre de minorer de 1 à $2 \%$ les émissions de GES.

\section{6 / Stockage de carbone}

La séquestration du carbone représente un potentiel important d'atténuation des émissions de GES des systèmes d'élevage herbivore (Soussana et al 2010). Au-delà de la préservation du stock de carbone présent dans les sols, des pratiques de gestion des prairies peuvent avoir un effet sur les conditions physico-chimiques du milieu, la protection et l'augmentation de la matière organique présente dans le sol. Ainsi, l'apport modéré d'azote (engrais, déjections) accroît la séquestration du carbone; a contrario, une carence en azote peut provoquer un déstockage de carbone car les microbes du sol puisent dans les réserves humiques du sol (Fontaine et al 2004, Klumpp et al 2009). La nature du couvert végétal influence également la capacité d'un agrosystème à séquestrer du carbone (Loiseau et al 2001). La présence de légumineuses permet une autorégulation en azote et donc le maintien du stock de carbone (Loiseau et al 2001, Soussana et al 2010). Le pâturage permet un meilleur stockage du carbone que la fauche via un apport direct de matière organique par les déjections et une moindre exportation de carbone du fait de l'herbe résiduelle (Reeder et Schuman 2002, Soussana et al 2007 et 2010). Inversement, la fauche exclusive et fréquente de la prairie (Klumpp et al 2007) peut conduire à des niveaux de prélèvement trop forts (exportations de carbone importantes et répétées) et à un déstockage. Enfin, l'intensité du pâturage joue également sur les niveaux de stockage, en lien avec l'herbe résiduelle. Les pâturages très ras laissent moins d'organes aériens, sources de litière puis de carbone, et le surpâturage peut engendrer une dégradation du couvert végétal (Jones et Donelly 2004). Inversement, les pâturages moins sévères laissent davantage d'organes sénescents (Louault et al 2005) favorables au stockage de carbone. Enfin, l'augmentation de la durée de la rotation des prairies temporaires ou la conversion de prairies temporaires en prairies permanentes augmente le stockage de carbone. Pour ces différentes pratiques, réduction des apports d'engrais azotés sur des parcelles intensives, conversion des prairies de graminées en mélange de graminées et légumineuses, intensification modérée des prairies pauvres, le flux annuel de carbone stocké est compris entre 200 et $500 \mathrm{~kg}$ $\mathrm{C} /$ ha (Soussana et al 2010). Sur la base de ces pratiques favorables ou défavorables au stockage de carbone, il reste à établir le meilleur compromis entre performances animales et stockage de carbone par les prairies.

Par ailleurs, la préservation et l'implantation de haies associées aux prairies constituent aussi un potentiel important de stockage de carbone dans les sols sur de longues durées. Au niveau des cultures, les principaux facteurs de variation concernent la diminution du travail du sol, voire un passage au non-travail, qui limite le phénomène de déstockage de carbone (l'effet bénéfique du passage au non-labour est toutefois réversible). Par ailleurs, la restitution au sol des résidus de la culture ou l'épandage de déchets organiques, de même que l'implantation de cultures intermédiaires pour éviter les sols nus (avec non-exportation des résidus) accroissent les apports de matières organiques et sont favorables au stockage de carbone.

Globalement, une modification de pratiques ou une évolution des surfaces en prairies au sein des exploitations permet une réduction de l'empreinte carbone comprise entre 3 et $10 \%$ selon le système (tableau 8).

\section{Conclusion}

L'élevage bovin contribue fortement aux émissions de GES de l'agriculture. Evaluer précisément ses émissions, puis construire un plan d'action est nécessaire pour réduire son impact sur le changement climatique. Toutefois, il faut rappeler que l'élevage de ruminants possède la particularité de pouvoir compenser en partie les émissions de GES grâce au stockage de carbone associé aux prairies et aux infrastructures agroécologiques (haies, bosquets...). Il est ainsi admis que les sols représentent le puits de carbone le plus important sur terre (1500 milliards de tonnes de carbone, GIEC 2007) et que la gestion optimale des prairies constitue une voie prometteuse pour réduire les émissions de GES (Arrouays et al 2002, FAO 2010, Soussana et al 2010).

L'analyse globale à l'échelle du système d'élevage est cruciale afin de réaliser une évaluation exhaustive des émissions de GES du secteur et de l'empreinte carbone des produits agricoles au portail de l'exploitation. Les mises au point méthodologiques en cours devront permettre à terme une détermination harmonisée des impacts. D'importants échanges nationaux et internationaux seront encore nécessaires dans les années à venir afin d'affiner ces méthodologies et les référentiels associés. Les évaluations conduites sur les systèmes de production français et étrangers montrent une variabilité de l'empreinte carbone inter-système, mais encore plus nettement une variabilité importante intra-système qui dépend du niveau d'optimisation des pratiques. Le différentiel observé peut ainsi atteindre jusqu'à $30 \%$ entre les exploitations optimisées et les systèmes non optimisés. Ce constat met en évidence les marges de progrès envisageables pour certaines exploitations grâce à une optimisation des pratiques.

Parallèlement un nombre important de leviers modulant les émissions est identifié. Les émissions évitées grâce à l'application de ces leviers varient de 0 à $10 \%$ (tableau 8). Certains leviers nécessitent des travaux de recherche complémentaires afin d'en préciser leur efficacité, leur faisabilité et leur coût. D'autres, liés à des pratiques éprouvées sont aujourd'hui disponibles et applicables en exploitation. Leur mise en œuvre peut dans certains cas se traduire par des économies sur le fonctionnement de l'exploitation ou alors nécessiter des investissements parfois importants. Globalement pour les filières bovines, un plan d'action qui rassemblerait plusieurs pistes de réduction compatibles laisse entrevoir un potentiel 
de réduction des émissions de GES compris entre 5 et $15 \%$ pour des exploitations dont l'empreinte carbone se situe aujourd'hui dans la moyenne observée. Ces potentiels de réduction mettent en avant la faculté de l'élevage de répondre au défi du changement climatique. Il faut de plus avoir à l'esprit que les objectifs de réduction de 20,30\%, voire davantage sont difficilement atteignables en l'état actuel des connaissances, mais que les avancées technologiques, la recherche en génétique etc., devraient permettre d'y répondre à terme.

D'ores et déjà, il faut préciser que l'ensemble des investigations conduites en matière d'évaluation des émissions de GES et de recherche de moyens d'atténuation permettent :

- d'apporter aux éleveurs de bovins et à leur environnement technique et administratif les connaissances, outils et méthodes permettant d'orienter leurs itinéraires techniques, voire de modifier leurs systèmes de production dans un objectif de réduction des émissions de GES et d'augmentation du stockage de carbone,

- de préciser aux éleveurs de bovins les incidences économiques, positives ou négatives, générées par l'application des leviers d'action dans les exploitations,

- d'indiquer aux filières et aux industriels du lait et de la viande bovine l'empreinte carbone atteignable au portail de l'exploitation,
- d'apporter à la France la possibilité de réduire son impact GES associé à l'activité d'élevage bovin.

Enfin, l'analyse de l'impact environnemental de l'activité d'élevage ne doit pas se limiter au seul impact GES. Sans remettre en cause la nécessité et l'urgence de mettre en place des plans d'action visant à limiter les émissions de GES, une approche monocritère n'apparaît pas pertinente et peut se traduire par des transferts de pollution (pollution des eaux, acidification atmosphérique ...). De plus, les systèmes d'élevages de ruminants présentent d'autres atouts, comme la contribution au maintien de la biodiversité qui nécessite d'être intégrée dans l'élaboration de plans d'actions pour les filières.

\section{Références}

Alford A.R., Hegarty R.S., Parnell P.F., Cacho O.J., Herd R.M., Griffith G.R., 2006. The impact of breeding to reduce residual feed intake on enteric methane emission from the Australian beef industry. Aust. J. Exp. Agric., $46,813-820$

Arrouays D., Balesdent J., Germon J.C., Jayet P.A., Soussana J.F., Stengel P., (Eds), 2002. Contribution à la lutte contre l'effet de serre. Stocker du carbone dans les sols agricoles de France? Expertise scientifique collective, INRA, synthèse, 36p.

Basset-Mens C., Ledgard S., Carran A., 2005. First life cycle assessment of milk production from New Zealand dairy farm systems. Anzsee conf., 258-265.

Basset-Mens C., Ledgard S., Boyes M., 2007. Eco-efficiency of intensification scenarios for milk production in New Zealand. Ecol. Econom., 68, 1615-1625.

Bellamy P.H., Loveland P.J., Bradley R.I., Lark R.M., Kirk G.J.D., 2005. Carbon losses from all soils across England and Wales 19782003. Nature, 437, 245-248.

Benchaar C., Pomar C., Chiquette J., 2001. Evaluation of dietary strategies to reduce methane production in ruminants: a modeling approach. Can. J. Anim. Sci., 81, 563-574.

Blanchin J.Y., 2010. Energie et gaz à effet de serre liés à la construction des bâtiments d'élevage. Institut de l'Elevage, Paris, France, 8p.

Casey J.W., Holden N.M., 2006. Quantification of GHG emissions from suckerbeef production in Ireland. Agric. Syst., 90, 7998.

Cederberg C., Mattsson B., 2000. Life cycle assessment of milk production. A comparison of conventional and organic farming. J. Clean. Prod., 8, 49-60.

Cederberg C., Darelius K., 2002. Using LCA methodology to assess the potential environmental impact of intensive beef and pork production. Dep. Appl. Environ. Sci., Gotëborg University, Sweden.

Cederberg C., Stadig M., 2003. System expansion and allocation in life cycle assessment of milk and beef production. Int. J. LCA., 350-356.
Cederberg C. Flysjö A., 2004. Life Cycle inventory of 23 dairy farms in South-Western Sweden, Sik rapport, 728, 63p.

Chambaut H., Moussel E., Pavie J., Contard J.P., Galisson B., Fiorelli J.L., Leroyer J., 2011. Profils environnementaux des exploitations d'élevage lait et viande en agriculture biologique et conventionnelle : enseignements du projet CedABio, $4 \mathrm{p}$.

Ciais P., Reichstein M., Viovy N., Granier A., Ogee J., Allard V., Aubinet M., Buchmann N., Bernhofer C., Carrara A., Chevallier F., De Noblet N., Friend A.D., Friedlingstein P., Grunwald T., Heinesch B., Keronen P., Knohl A., Krinner G., Loustau D., Manca G., Matteucci G., Miglietta F., Ourcival J.M., Papale D., Pilegaard K., Rambal S., Seufert G., Soussana J.F., Sanz M.J., Schulze E.D., Vesala T., Valentini R., 2005. Europe-wide reduction in primary productivity caused by the heat and drought in 2003. Nature, 437, 529-533.

Corré W.J., 2002. Agricultural land use and emissions of methane and nitrous oxide in Europe. Plant Res. Int., Wageningen. The Netherlands, 28p.

Cruickshank G., Thomson B., Muir P., 2009. Effect of management change on methane output within a sheep flock. Proc. N.Z. Soc. Anim. Prod., 69, 170-173.

Da Silva V.P., Van Der Werf H.M.G., Spies A., Roberto Soares S., 2010. Variability in environnemental impacts of Barazilian soybean according to crop production and transport scenarios. J. Environ. Manag., 91, 1831-1839.

De Klein C.A.M., Eckard R.J., 2008. Targeted technologies for nitrous oxide abatement from animal agriculture. Austr. J. Exp. Agric., 48, 14-20.

De Vries M., De Boer I.J.M., 2010. Comparing environmental impacts for livestock products: A review of cycle assessments. Livest. Sci., 120, 1-11.

Di H.J., Cameron K.C., 2010. An effective mitigation technology for reducing nitrous oxide emissions from grazed grassland. Banff, Canada. Greenhouse Gases Anim. Agric. Conf., October 3-8.

Dollé J.B., Gac A., Le Gall A., 2009 L'empreinte carbone du lait et de la viande bovine. Renc. Rech. Ruminants, 16, 233-236.

Dollé J.B., Manneville V., Gac A., Charpiot A., 2011. Emissions de gaz à effet de serre et consommations d'énergie des viandes bovines et ovines françaises : revue bibliographique et évaluations sur l'amont agricole, Institut de l'Elevage, Paris, France, 59 p.

Doreau M., Dollé J.B., 2011a. Strategies for reducing greenhouse gas emissions in dairy production. A European perspective. Proc. Eastern Nutr. Conf., Montréal, Canada, 12-13 avril 2011, 57-77.

Doreau M., Van Der Werf H.M.G., Micol D., Dubroeucq H., Agabriel J., Rochette Y., 2011b. Enteric methane production and greenhouse gases balance of diets differing in concentrate in fattening phase of a beef production system. J. Anim. Sci., 89, 2518-2528.

Erzinger S., Dux D., Zimmermann A., Badertscher Fawaz R., 2003. LCA of animal products from different housing systems in Switzerland: relevance of feedstuffs, infrastructure and energy use. In: Life cycle assessment in the agri-food sector. Halberg N. (Ed.). Danish Inst. Agric. Sci., Bygholm, Denmark, 55-63.

FAO, 2006. Livestock's Long Shadow: environmental issues and options. FAO, Rome, Italy, 36p.

FAO, 2010. Greenhouse gas emissions from the dairy sector: A life cycle assessment. FAO, Rome, Italy, 97p.

FIL, 2010. A common carbon footprint approach for dairy, Bulletin IDF, 46p.

Fontaine S., Bardoux G., Abbadie L. Mariotti A., 2004. Carbon input to soil may decrease soil carbon content. Ecol. Letters, 7, 314-320.

Gac A., Deltour L., Cariolle M., Dollé J.B., Espagnol S., Flénet F., Guingand N., Lagadec S., Le Gall A., Lellahi A., Malaval C., Ponchant P., Tailleur A., 2010a. GES'TIM, Guide méthodologique pour l'estimation des impacts des activités agricoles sur l'effet de serre. Version 1.2., Institut de l'Elevage, Paris, France, 156p.

Gac A., Dollé J.B., Le Gall A., Klumpp K., Tallec T., Mousset J., Eglin T., Bispo A., 
2010b. Le stockage de carbone par les prairies. Institut de l'Elevage, Paris, France, 12p.

Gac A., Manneville V., Raison C., Charroin T., Ferrand M., 2010c. L'empreinte carbone des élevages d'herbivores : présentation de la méthodologie d'évaluation appliquée à des élevages spécialisés lait et viande. Renc. Rech. Rum., 17, 335-342.

Gerber P., Vellinga T., Opio C., Steinfeld H., 2011. Productivity gains and greenhouse gas emissions intensity in dairy systems. Livest. Sci., doi:10.1016.j.livsci.2011.03.012.

Haas G., Wetterich F., Köpke U., 2001. Comparative intensive, extensified and organic grassland farming in southern Germany by process life cycle assessment. Agric. Ecosyst. Env., 83, 43-53.

Hegarty R., 2004. Genotype differences and their impact on digestive tract function of ruminants: a review. Austr. J. Expl. Agric., 44, 459467.

Henriksson M., Flysjö A., Cederberg C., Swensson C., 2011. Variation in carbon footprint of milk due to management differences between Swedish dairy farms. Animal, 5, published online, doi:10.1017/S1751731111000 437.

Hindrichsen I.K., Wettstein H.R., Machmuller A., Kreuzer M., 2006. Methane emission, nutrient degradation and nitrogen turnover in dairy cows and their slurry at different milk production scenarios with and without concentrate supplementation. Agric. Ecosyst. Environ., 113, 150-161.

IPCC, 2006. IPCC Guidelines for National Greenhouse Gas Inventories. Intergovernmental panel on climate change. Nat. Greenhouse Gas Inv. Ref. Manual, 4

Jones M.B., Donnelly A., 2004. Carbon sequestration in temperate grassland ecosystems and the influence of management, climate and elevated $\mathrm{CO}_{2}$. New Phytol., 164, 423-439.

Kebreab E., France J., Beever D.E., Castillo A.R., 2001. Nitrogen pollution by dairy cows and its mitigation by dietary manipulation. Nutr. Cycl. Agroecosyst., 60, 275-285.

Klumpp K., Soussana, J.F., Falcimagne R. 2007. Effects of past and current disturbance on carbon cycling in grassland mesocosms. Agric. Ecosyst. Env., 121, 59-73.

Klumpp K., Fontaine S., Attard E., Gleixner G., Leroux X., Soussana J.F., 2009. How land use change shifts C-poor, productive ecosystems to C-rich, unproductive ecosystems and vice versa. J. Ecol., 91, 867-885.

Kristensen T., Mogensen L., Knudsen M.T., Hermansen J.E., 2011. Effect of production system and farming strategy on greenhouse gas emissions from commercial dairy farms in a life cycle approach. Livest. Sci., 13p.

Ledgard S., Schils R., Eriksen J., Luo J., 2009. Environmental impacts of grazed clover/grass pastures. Ir. J. Agric. Food Res., 48, 209-226.

Le Gall A., Beguin E., Dollé J.B., Manneville V., Pflimlin A., 2009. Nouveaux compromis techniques pour concilier les impératifs d'efficacité économique et environnementale des systèmes d'élevage herbivore. Proc. Journ. AFPF, 15p

Leip A., Weiss F., Wassenaar T., Perez I., Fellmann T., Loudjani P., Tubiello F., Grandgirard D., Monni S., Biala K., 2010.
Evaluation of the livestock sector's contribution to the EU greenhouse gas emission (GGELS), final report. Europ. Comm. Joint Res. Centre, 32p.

Loiseau P., Soussana J.F., Louault F., Delpy R., 2001. Soil N contributes to the oscillations of the white clover content in mixed swards under simulated grazing (Lolium perenne $\mathrm{L}$, Trifolium repens L.). Grass Forage Sci., 56, 205-217.

Louault F., Pillar V.D., Aufrere J., Garnier E., Soussana J.F., 2005. Plant traits and function types in response to reduced disturbance in semi-natural grassland. J. Veget. Sci., 16, 151160

Lovett D., Stack L., Lovell S., Callan J., Flynn B., Hawkins M., O’Mara F., 2005. Manipulating enteric methane emissions and animal performance of late-lactation dairy cows through concentrate supplementation at pasture. J. Dairy Sci., 88, 2836-2842.

Lovett D.K., Shalloo L., Dillon P., O’Mara F.P., 2006. A systems approach to quantify greenhouse gas fluxes from pastoral dairy production as affected by management regime. Agric. Syst., 88, 156-179.

Luo J., de Klein C.A.M., Ledgard S.F., Saggar S., 2010. Management options to reduce nitrous oxide emissions from intensivey grazed pastures: A review. Agric. Ecosys. Env., 136, 282-291.

Mac Donald K.A., Williams Y., Dobson-Hil B., 2010. Effectiveness of a nitrification inhibitor (DCn) on a Coastal Taranaki dairy farm. Proc. N.Z. Grass Assoc., 72, 147-152.

Martin C., Morgavi D.P., Doreau M., 2010. Methane mitigation in ruminants: from microbe to the farm scale. Animal, 4, 351-365.

Martin C., Pomiès D., Ferlay A., Eugène M., Martin B., Doreau M., Chilliard Y., 2010. Methane output in dairy cows in response to long-term feeding of grass-based diets supplemented with linseed or rapeseed. McGeough E.J., McGinn S.M. (Eds). Banff, Canada, Proc Greenhouse Gases Anim. Agric., Conf., 134 135

Martin C., Pomiès D., Ferlay A., Rochette Y., Martin B., Chilliard Y., Morgavi D.P. Doreau M., 2011. Methane output and rumen microbiota in dairy cows in response to long term supplementation with linseed or rapeseed of grass silage or pasture based diets. Proc. N.Z. Soc. Anim. Prod., 71, 243-247.

Metz B., Davidson O.R., Bosch P.R., Dave R., Meyer L.A., 2007. Contribution of working group III to the fourth assessment report of the intergovernmental panel on climate change. Cambr. Univ. Press, Cambridge, United Kingdom and New York, USA, 70p.

Monaghan R.M, Hedley M.J., Di H.J., McDowell R.W., Cameron K.C., Ledgard S. 2007. Nutrient management in New Zeland pastures. Recent developements and future issues. N.Z. J. Agric. Res., 50, 181-201.

Monaghan R.M, Smith L.C, Ledgard S.F., 2009. The effectiveness of a granular formulation of dicyandiamide (DCD) in limiting nitrate leaching from a grazed dairy pasture. N.Z. J. Agric. Res., 2, 145-159.

Morgan J.A., Pataki D.E., Korner C., Clark H., Del Grosso S.J., Grunzweig J.M., Knapp A.K., Mosier A.R., Newton P.C.D., Niklaus P.A., Nippert J.B., Nowak R.S., Parton W.J. Polley H.W., Shaw M.R., 2004. Water relations in grassland and desert ecosystems exposed to elevated atmospheric $\mathrm{CO}_{2}$. Oecologia, 140, 11-25.

Nielsen P.H., Nielsen A.M., Weidema B.P., Dalgaard R., Halberg N., 2003. LCA food data base. http://www.lcafood.dk/

Pelletier N., Pirog R. and Rasmussen R., 2010. Comparative life cycle environmental impacts of three beef production strategies in the Upper Midwestern United States. Agric. Syst., 103, 380-389.

Phetteplace H., Johnson D., Seidl A., 2001. Greenhouse gas emissions from simulated beef and dairy livestock systems in the United States. Nut. Cycl. Agroecosys., 60, 99-102.

Reeder J.D., Schuman G.E., 2002. Influence of livestock grazing on $\mathrm{C}$ sequestration in semi-arid mixed-grass and short-grass rangelands. Environ Pollut., 116, 457-463.

Rossier D., Gaillard G., 2001. Bilan écologique de l'exploitation agricole, méthode et application à 50 entreprises. Office Fédéral de l'Agriculture, $163 \mathrm{p}$.

Schils R.L.M., Verhagen A., Aarts H.F.M., Sebek L.B.J., 2005. A farm level approach to define successful mitigation strategies for GHG emissions from ruminant livestock systems. Nutr. Cycl. Agroecosys., 71, 163-175.

Schulze E.D., Luyssaert S., Ciais P., Freibauer A., Janssens I.A., Soussana J.F., Smith P., Grace J., Levin I., Thiruchittampalam B., Heimann M., Dolman A.J., Valentini R., Bousquet P., Peylin P., Peters W., Rodenbeck C., Etiope G., Vuichard N., Wattenbach M., Nabuurs G.J., Poussi Z., Nieschulze J., Gash J.H., 2009. Importance of methane and nitrous oxide for Europe's terrestrial greenhouse-gas balance. Nature Geosci., 2, 842-850

Smith P., Chapman S.J., Scott W.A., Black H.I.J., Wattenbach M., Milne R., Campbell C.D., Lilly A., Ostle N., Levy P.E., Lumsdon D.G., Millard P., Towers W., Zaehle Z., Smith J.U., 2007. Climate change cannot be entirely responsible for soil carbon loss observed in England and Wales, 1978-2003. Global Change Biol., 13, 2605-2609.

Soussana J.F., Hartwig U.A., 1996. The effects of elevated $\mathrm{CO}_{2}$ on symbiotic $\mathrm{N}_{2}$ fixation : a link between the carbon and nitrogen cycles in grassland ecosystems. Plant and Soil, 187, 321-332.

Soussana J.F., Lüscher A., 2007. Temperate grasslands and global atmospheric change: a review. Grass Forage Sci., 62,127-134.

Soussana J.F., Allard V., Pilegaard K., Ambus P., Amman C., Campbell C., Ceschia E., Clifton-Brown J., Czobel S., Domingues R., Flechard C., Fuhrer J., Hensen A., Horvath L., Jones M., Kaspe G., Martin C., Nagy Z., Neftel A., Raschi A., Baronti S., Rees R.M. Skiba U., Stefani P., Manca G., Sutton M., Tuba Z., Valentini R., 2007. Full accounting of the greenhouse gas $\left(\mathrm{CO}_{2}, \mathrm{~N}_{2} \mathrm{O}, \mathrm{CH}_{4}\right)$ budget of nine European grassland sites. Agric. Ecosyst. Env., 121, 121-134.

Soussana J.F., Tallec T., Blanfort V., 2010 Mitigating the greenhouse gas balance of ruminant production systems through carbon sequestration in grasslands. Animal, 4, 334350 .

Tomasula P.M., Nutter D.W., 2011 Mitigation of greenhouse gas emissions in the production of fluid milk. Food Nutr. Res., 62, 42-88. 
Tubiello F., Soussana J.F., Howden S.M., Easterling W., 2007. Crop and pasture response to climate change. Proc. Nat. Acad. Sci. U.S.A, 104, 19686-19690

Van der Werf H.M.G., Kanyarushoki C., Corson M.S., 2009. An operational method for the evaluation of resource use and environmental impacts of dairy farms by life cycle assessment. J. Env. Manag., 90, 3643-3652.

Vellinga T, de Haan M.H.A., Schils R.L.M., Evers A., van den Pol-van Dasselaar A., 2011. Implementation of GHG mitigation on intensive dairy farms: farmers preferences and variation in cost effectiveness. Livest. Sci., 137, 185-195.
Vérité R., Faverdin P., Agabriel J., 1997. In: Recent advances in animal nutrition. Wiseman J. Garnsworthy P.C. (Eds). Nottingham Univ. Press, Nottingham, UK, 153-166.

Veysset P., Lherm M., Bébin D., 2010. Energy consumption, greenhouse gas emissions and economic performance assessments in French Charolais suckler cattle farms Model-based analysis and forecasts. Agric. Syst., 103, 41-50.

Veysset P., Lherm M., Bébin D., 2011. Productive, environmental and economic performances assessments of organic and conventional suckler cattle farming systems. Org. Agric., 1-16.
Watson R., Noble I., Bolin B., Ravindranath N., Verardo D., Dokken D., 2002. Land Use, Land Use Change, and Forestry. Camb. Univ. Press, Cambridge, UK.

Woodward S.L., Waghorn G.C., Thomson N.A., 2006. Supplementing dairy cows with oils to improve performance and reduce methane - does it work ? Proc. N.Z. Soc. Anim. Prod., 66, 176-181.

Zehetmeier M., Baudracco J., Hoffmann H., HeiBenhuber A., 2011. Does increasing milk yield per cow reduce greenhouse gas emissions? A system approach. Animal, 13p.

\section{Résumé}

Le contexte environnemental actuel, tant politique (objectifs de réduction des émissions de gaz à effet de serre) que sociétal (information du consommateur), nécessite de préciser les impacts de l'activité d'élevage bovin en matière de changement climatique. $L$ 'enjeu est de connaître précisément les niveaux d'émissions de gaz à effet de serre (GES) et de stockage de carbone, des différents modes de production. Pour cela, une évaluation basée sur la méthodologie de l'Analyse du Cycle de Vie (ACV) est mise au point à l'échelle du système d'élevage. Cette approche permet d'avoir une vision globale de l'activité d'élevage intégrant l'ensemble des processus internes et externes au fonctionnement de l'exploitation. Ainsi pour les systèmes laitiers français, l'empreinte carbone brute du lait est en moyenne de $1,26 \mathrm{~kg} \mathrm{CO} / \mathrm{kg}$ de lait. La prise en compte du stockage de carbone sous les prairies et les haies se traduit par une compensation comprise entre 6 et $\mathbf{4 3 \%}$ selon les systèmes, en fonction de la part de prairies. L'empreinte carbone nette du lait français est alors en moyenne de $1,0 \mathrm{~kg} \mathrm{CO} / / \mathrm{kg}$ de lait. Dans les systèmes bovins viande français, l'empreinte carbone brute est comprise entre 14,8 et $16,5 \mathrm{~kg} \mathrm{CO} / \mathrm{kg}$ viande vive en fonction du système de production (naisseur vs naisseur/engraisseur). Après prise en compte du stockage de carbone qui permet une compensation comprise entre 24 et $53 \%$, l'empreinte carbone nette est comprise entre 7,9 et $11,3 \mathrm{~kg} \mathrm{CO} / \mathrm{kg}$ viande vive. De nombreux leviers d'action sont identifiés dans les systèmes d'élevage de ruminants pour réduire l'empreinte carbone des produits au portail de la ferme. Certains concernent une optimisation des systèmes de production (ajustement des apports alimentaires, gestion de la fertilisation...) et se traduisent par des économies en matière d'intrants. D'autres nécessitent la mise en place de nouvelles technologies et se traduiront donc par un investissement ou un coût de fonctionnement supérieur aux schémas actuels de production.

\section{Abstract}

\section{Greenhouse gases in cattle breeding : evaluation and mitigation strategies}

In today's environmental context, as much political (the reduction of greenhouse gas emissions) as social (consumer demands for information concerning food products), there is a need to determine the influence of ruminant livestock on climate change. It has become crucial to quantify precisely the levels of greenhouse gas emissions (GHG) and carbon sequestration for different ruminant livestock systems by using Life Cycle Assessment (LCA) to calculate the carbon footprint of dairy and beef farms. This approach allows us to account for GHG in relation with direct and indirect emissions. Thus in the French dairy system, the average gross carbon footprint is $1.26 \mathrm{~kg} \mathrm{CO} / \mathrm{kg}$ milk. Carbon sequestration under grassland and hedges compensates for GHG emissions ranging from 6 to $43 \%$ according to the system utilized. Consequently the net carbon footprint is $1.0 \mathrm{~kg} \mathrm{CO}_{2} / \mathrm{kg}$ milk. In the French beef system, the gross carbon footprint for raw materials is comprised between 14.8 and $16.5 \mathrm{~kg} \mathrm{CO} / \mathrm{kg}$ of live meat depending on the production system. It must be mentioned that carbon sequestration represents compensation in a range of 24 to $53 \%$ and the net carbon footprint is between 7.9 and $11.3 \mathrm{~kg} \mathrm{CO} / \mathrm{kg}$ of live meat. These investigations demonstrate that numerous mitigation actions have been identified in the livestock systems to reduce the carbon footprint of milk and meat at the farm gate. Some of them concern management practices (adjustment of dietary intake, fertilization management...) which result in substantial savings in agricultural expenses. Others require the installation of new technologies which would require additional funds to improve the production processes.

DOLLÉ J.-B., AGABRIEL J., PEYRAUD J.-L., FAVERDIN P., MANNEVILLE V., RAISON C., GAC A., LE GALL A., 2011. Les gaz à effet de serre en élevage bovin : évaluation et leviers d'action. In : Gaz à effet de serre en élevage bovin : le méthane. Doreau M., Baumont R., Perez J.M. (Eds). Dossier, INRA Prod. Anim. 24, 415-432. 
